\title{
Investigation of Connecting Wind Turbine to Radial Distribution System on Voltage Stability Using SI Index and $\lambda-V$ Curves
}

\author{
Gamal Abd El-Azeem Mahmoud, Eyad Saeed Soliman Oda \\ Department of Electrical Engineering, Faculty of Engineering, Suez Canal University, Ismailia, Egypt \\ Email: Eng.Eyad.S.S.Oda@gmail.com
}

Received 27 November 2015; accepted 25 January 2016; published 28 January 2016

Copyright (C) 2016 by authors and Scientific Research Publishing Inc.

This work is licensed under the Creative Commons Attribution International License (CC BY).

http://creativecommons.org/licenses/by/4.0/

c) (i) Open Access

\begin{abstract}
The growth of wind energy penetration level in distribution system raises the concern about its impact on the operation of the power system, especially voltage stability and power loss. Among the major concerns, this paper studied the impact of connecting wind Turbine (WT) in radial distribution system with different penetration levels and different power factor (lead and lag) on power system voltage stability and power loss reduction. Load flow calculation was carried out using forward-backward sweep method. The analysis proceeds on 9- and 33-bus radial distribution systems. Results show that voltage stability enhancement and power loss reduction should be considered as WT installation objective.
\end{abstract}

\section{Keywords}

Power Loss, Radial Distribution System, Si Index, Voltage Stability, Optimal Size and Location of Wind Turbine

\section{Introduction}

In the past few years, great progress has been achieved in wind energy (WE) field as distributed generation source (DGS) because wind energy has a lot of merits such as environment, economic and technical. Environmentally it's clean energy source and meets the requirement of reducing global warming. Technically the single wind turbine capable of generating power in few MW at present and in near future 2020 the rated power of WT may reach 10 - $20 \mathrm{MW}$ [1]. Economically it's the cheapest renewable energy source [1] [2], these merits make wind energy the most suitable source for future energy. Therefore, the penetration of wind turbines in electrical power systems will increase and they may begin to influence overall power system behavior, making it impossible 
to run a power system by only controlling large scale power plants. So it is important to study the behavior of wind turbines in an electrical power system. Refs [3]-[6] were concerned in impact of connecting distributed generation (DG) to the grid on voltage stability, to study the optimal penetration level of wind farms without compromising the system voltage stability in transmission level using energy storage systems studied in ref [7], while ref. [8] presented the impact of fixed speed WT on grid voltage stability in transmission level.

Distribution system (DS) is an important part of power system, and it represents the link between the bulk system and customers. It is estimated that as much as $13 \%$ of the total power generation is lost in the distribution networks. Approximately $70 \%$ of the total electric power system real power losses are associated with the distribution level [9]. Wind energy farms usually located at distribution area, so it's essential to study the behavior associated with connecting wind turbine generation units to distribution level.

Voltage stability and power losses problems are annoying problems in DS, voltage stability may spread to transmission system and cause blackout for the whole system. Extensive studies are needed to determine the best connection of WT to DSs with acceptable voltage level.

This paper is organized as follows. Section 2 describes load flow in distribution system. Section 3 describes the wind turbine penetration level. Section 4 describes the voltage stability index used to study voltage stability margin of the system. Section 5 describes power loss reduction. Section 6 describes optimization technique used to choose optimal size and location of WT. Results obtained considering voltage stability margin, voltage profile, and power loss are presented in Sections 7 and 8. Finally, Section 9 summarizes the main conclusions.

\section{Distribution System and Load Flow}

Power flow problems can be solved by several methods and are classified as either direct or iterative. The direct methods employ the direct solutions related to the linear system, but actually all methods are iterative because the basic problem involves the solution of a system of non-linear equations.

Although conventional power flow methods such as Newton-Raphson (NR) technique, Fast decoupled (FD) load flow and Gauss-Seidel are well developed in dealing with the transmission and sub-transmission sections of the power system networks, they are considered to be inefficient in handling distribution networks. This is because the (DS) is different in several ways from its transmission counterpart.

- DS has a strictly radial topology nature or weakly meshed networks in contrast with transmission systems which are "tightly" meshed networks.

- $\quad$ DS is a low voltage system having low $\mathrm{X} / \mathrm{R}$ ratio sections and a wide range of reactance and resistance values. The practical DSs low X/R ratio sections may cause both the NR and FD conventional methods to diverge. The line impedance angles are small enough to deteriorate the dominance of the NR Jacobian main diagonal, making it prone to singularity. Such a low $\mathrm{X} / \mathrm{R}$ value would also prevent the Jacobian matrix from being decoupled and simplified.

- DS may consist of a tremendously large number of sections and buses spread throughout the network.

All of these characteristics strongly suggest that DS is to be classified as an ill-conditioned power system.

In this paper a popular solution method used to treat ill conditioning is the forward-backward sweep (FB) method. The forward/backward method has been developed by D. Thukaram [10]; this method employs Ohm's law, Kirchhoff Voltage Law and Kirchhoff Current Law to calculate nodal voltages and branch currents.

The backward stage determines the branch currents from the end node towards the root node with a constant nodal voltage, while the forward stage is used to determine the nodal voltages by assuming the source nodal voltage is constant. The forward-backward substitution was replaced by the forward-backward sweep technique. The technique used in this paper is proposed in appendix A for more details refer to ref [11].

\section{Wind Turbine Penetration Level}

Wind turbine Penetration Level $(P L)$ used to study its impact on the power system operation. This $P L$ gives percentage WT generated power contribution in load supply is given by:

$$
P L=\frac{P_{W T}}{P_{l}} \times 100
$$

where 
$P_{W T}$ : wind turbine active power.

$P_{l}$ : total loadsactive power.

The paper studied the impact of WT at different $P L$ and different power factor (p.f) i.e. (lead and lag) on distribution system operation.

\section{Voltage Stability}

The voltage instability problem in a distribution network may spread to the transmission network causing a blackout as happened in Brazilian system in June 1997 [12], US/Canada blackout, the Scandinavian blackout and the Italian blackout, which all happened in 2003 [13]. It is attracting more attention from researchers around the world.

There are several definitions of voltage stability. One definition developed by IEEE and CIGRE that Voltage stability refers to the ability of a power system to maintain steady voltages at all buses in the system after being subjected to a disturbance from a given initial operating condition [14].

The term voltage collapse is also often used. It is the process by which the sequence of events accompanying voltage instability leads to a blackout or abnormally low voltages in a significant part of the power system.

\section{Voltage Stability Indices}

The main objective of Voltage Stability Indices (VSIs) is to estimate the distance from the current operating point to the system voltage marginally stable point. Numerical indices help operators to monitor how close the system is to collapse or to initiate automatic remedial action schemes to prevent voltage collapse.

Most of the VSIs that have been proposed are based on steady state power flow.

An index, which can be evaluated at all buses in radial distribution systems, was presented by M. Charkravorty and D. Das in [15].

The distribution system can be represented by the equivalent of two bus system as showing in Figure 1; the Equation represents the voltage stability index SI is given by:

$$
\begin{aligned}
S I(i+1)= & |V(i)|^{4}-4.0[P(i+1) \times(i)-Q(i+1) r(i)]^{2} \\
& -4.0[P(i+1) r(i)+Q(i+1) x(i)]|V(i)|^{2}
\end{aligned}
$$

where

$S I(i+1)$ : Voltage stability index of bus $i+1(i=1,2, \cdots, n)$.

$n$ : the total number of buses.

$i$ : the branch number.

$r$ (i): resistance of branch $i$.

$x(i)$ : reactance of branch $i$.

$V(i)$ : voltage of bus $i$.

$V(i+1)$ : voltage of bus $i+1$.

$P(i+1)$ : total real power load fed through bus $i+1$.

$Q(i+1)$ : total reactive power load fed through bus $i+1$.

When SI approaches $\mathbf{0 . 0}$, that means the system is unstable.

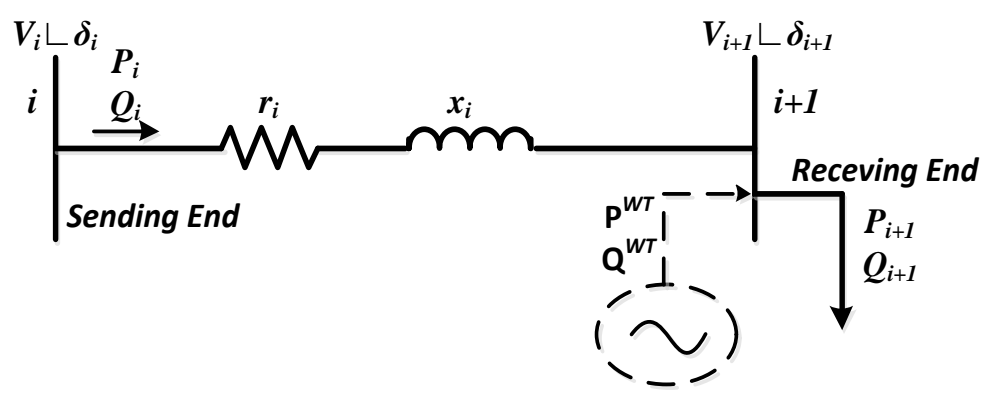

Figure 1. Simple two bus system. 


\section{Power Losses}

Before the WT connected to the system showing in Figure 1, the total active power losses are:

$$
\sum_{i=0}^{n} P_{\text {loss }_{I+1}}=\sum_{i=0}^{n}\left[\left(\frac{P_{i}^{2}+Q_{i}^{2}}{V_{i}^{2}}\right) \times r_{i}\right]
$$

Once the WT is connected at the system, three different situations may take place:

$$
\begin{array}{ll}
\text { - } & \text { situation 1: }\left(P^{W T}\right)_{i+1}<\left(\sum P_{\text {load }}\right)_{i+1} \\
\text { - } & \text { situation 2: }\left(P^{W T}\right)_{i+1}=\left(\sum P_{\text {load }}\right)_{i+1} \\
\text { - } & \text { situation 3: }\left(P^{W T}\right)_{i+1}>\left(\sum P_{\text {load }}\right)_{i+1}
\end{array}
$$

For situations 1 and 2, the injection of $P^{W T}$ reduces the $P_{i}$ drawn from bus $i$. In other words, the WT reduces the load's value at bus $i+1$. However, the WT in situation 3 acts as in situations 1 and 2, plus it will reverse a portion of the injected $P^{W T}$ back to bus $i$. Reducing the power loss and maintaining the voltage within acceptable limits are vital in WT applications. Although the first and second situations' results in some cases are significant, situation 3 leads to better results if the optimal WT size and location are employed.

To evaluate the impact of installing WT at distribution feeder on power loss reduction (PLR) the following relation used:

$$
P L R \%=\frac{P_{L O S S}-P_{L O S S}^{W T}}{P_{\text {LOSS }}} \times 100
$$

where

$P_{\text {LOSS }}:$ System losses without WT

$P_{L O S S}^{W T}:$ System loss after WT installation

\section{Optimal Size and Location of WT}

Optimal location and size of WT generation obtained using Grid Search Algorithm [16]-[18]. The Grid Search Algorithm is applied by adding WT to each bus, changing the size of WT from $0 \%$ of total load power to $100 \%$ of total load power with the step size of $0.05 \%$ of total load power. In this algorithm, the objective is to minimize total power losses of the system $\left(P_{\text {loss }}\right)$ by injected active power of $W T\left(P^{W T}\right)$ for WT placement The main constraints are to restrict the voltages along the radial system within $1 \pm 0.05 \mathrm{pu}$, as in Equation (5-a) and Equation (5-b).

To minimize $f\left(P^{W T}\right)=P_{\text {loss } T}$ subject to

$$
\begin{gathered}
\left|V_{i}\right| \leq 1 \pm 0.05 \text { p.u } \quad i=1,2, \cdots, n \\
0 \leq P^{W T} \leq \sum P_{\text {load }}
\end{gathered}
$$

The flowchart of the Grid Search algorithm to determine the optimum size and location of WT is proposed in the appendix B Figure B-1.

\section{First Case Study}

The first test case is a 9-bus [11] [19], single feeder, radial distribution system shown in Figure 2. This system has no laterals. The rated line voltage of the system is $23 \mathrm{kV}$. The details of the feeder and the load characteristics are given in Table $\mathrm{C}-1$ in Appendix C.

Using FB sweep analysis SI index and PLR are evaluated for different PL of WT.

Wind turbine installed with different penetration levels at bus 9 (weakest branch of distribution feeder based on the value of the VSI). It's found that as the penetration level increase system voltage stability enhanced and power loss decreased as shown in the following Figures 3-9. 


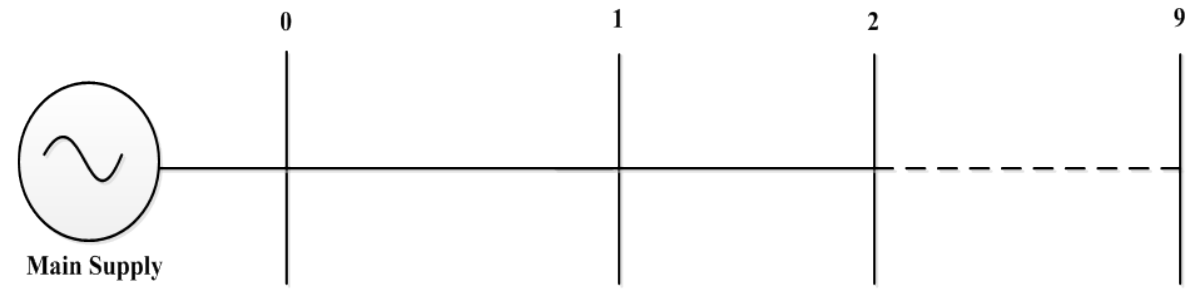

Figure 2. Single line diagram of 9-bus feeder.

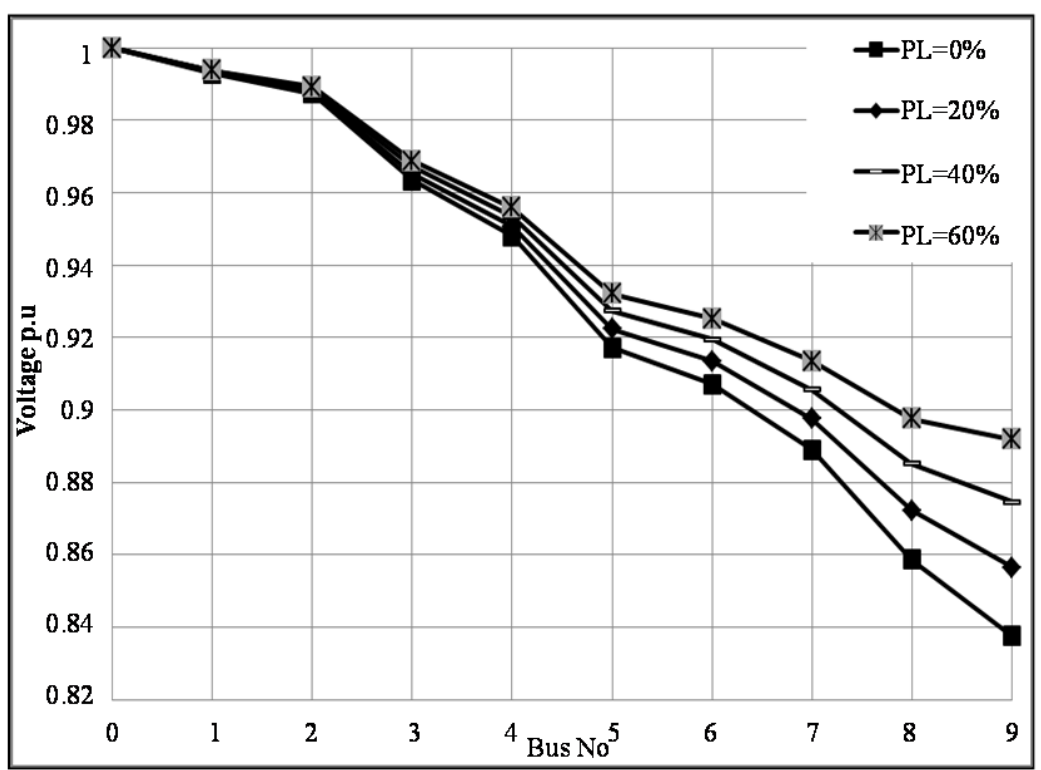

Figure 3. Bus voltages at different PLs (0.9 lead p.f.).

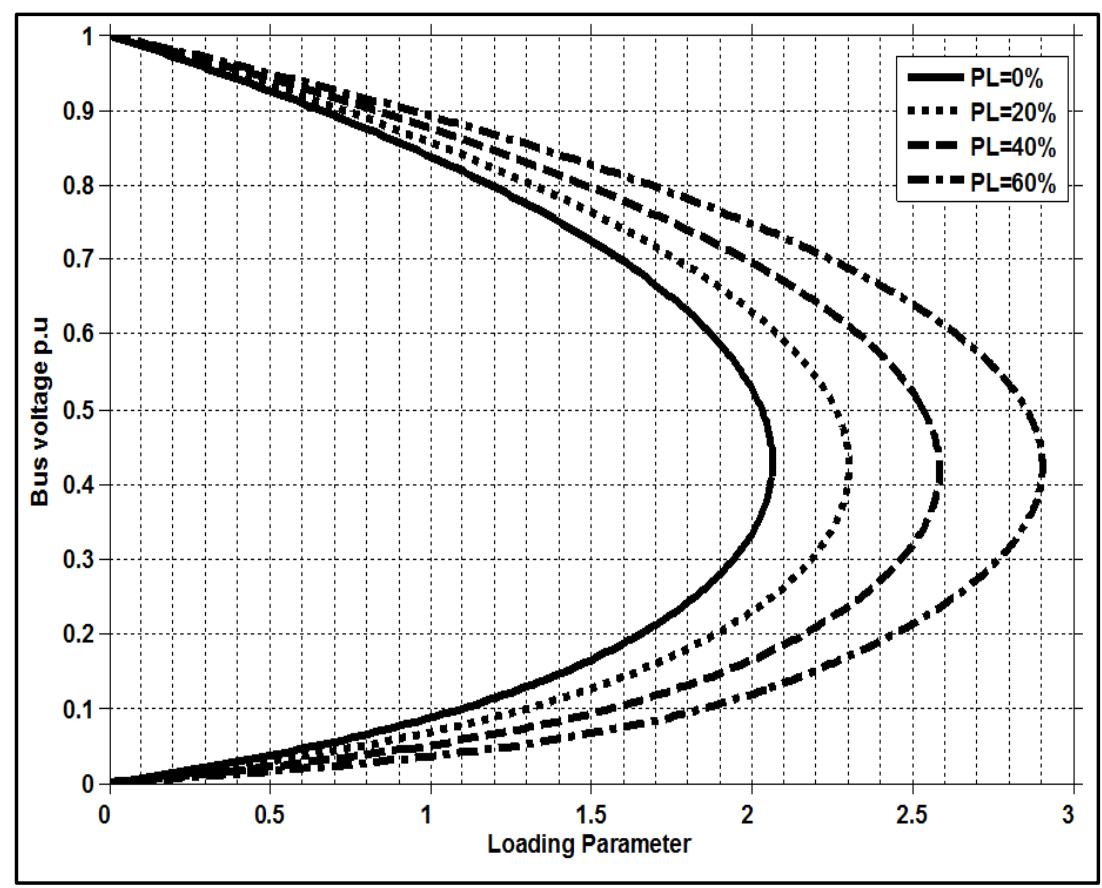

Figure 4. $\lambda-V$ curves at bus-9 with different PLs (lead p.f.). 


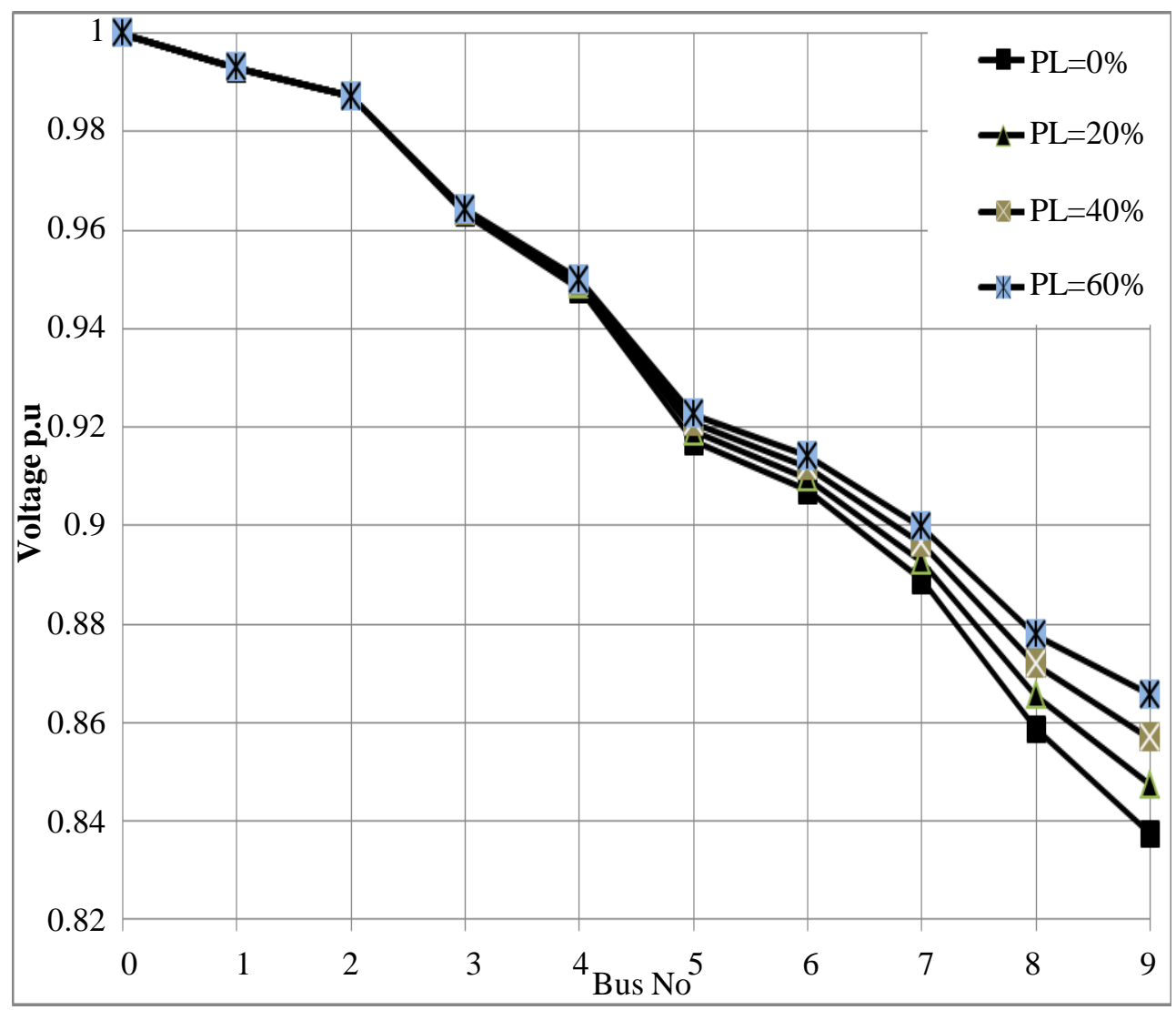

Figure 5. Bus voltages at different PLs (0.9 lag p.f.).

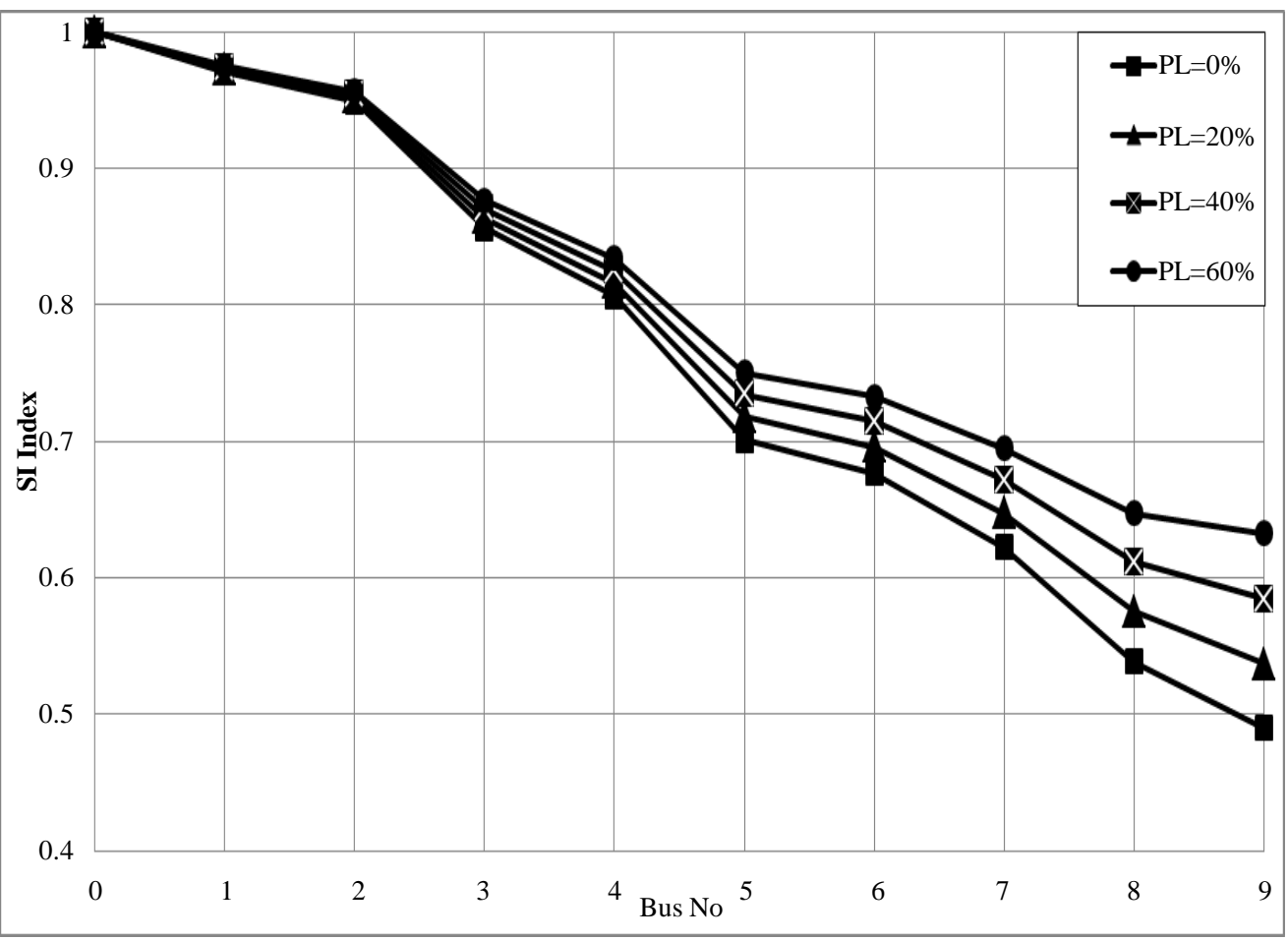

Figure 6. SI index at different PLs (0.9 lead p.f.). 


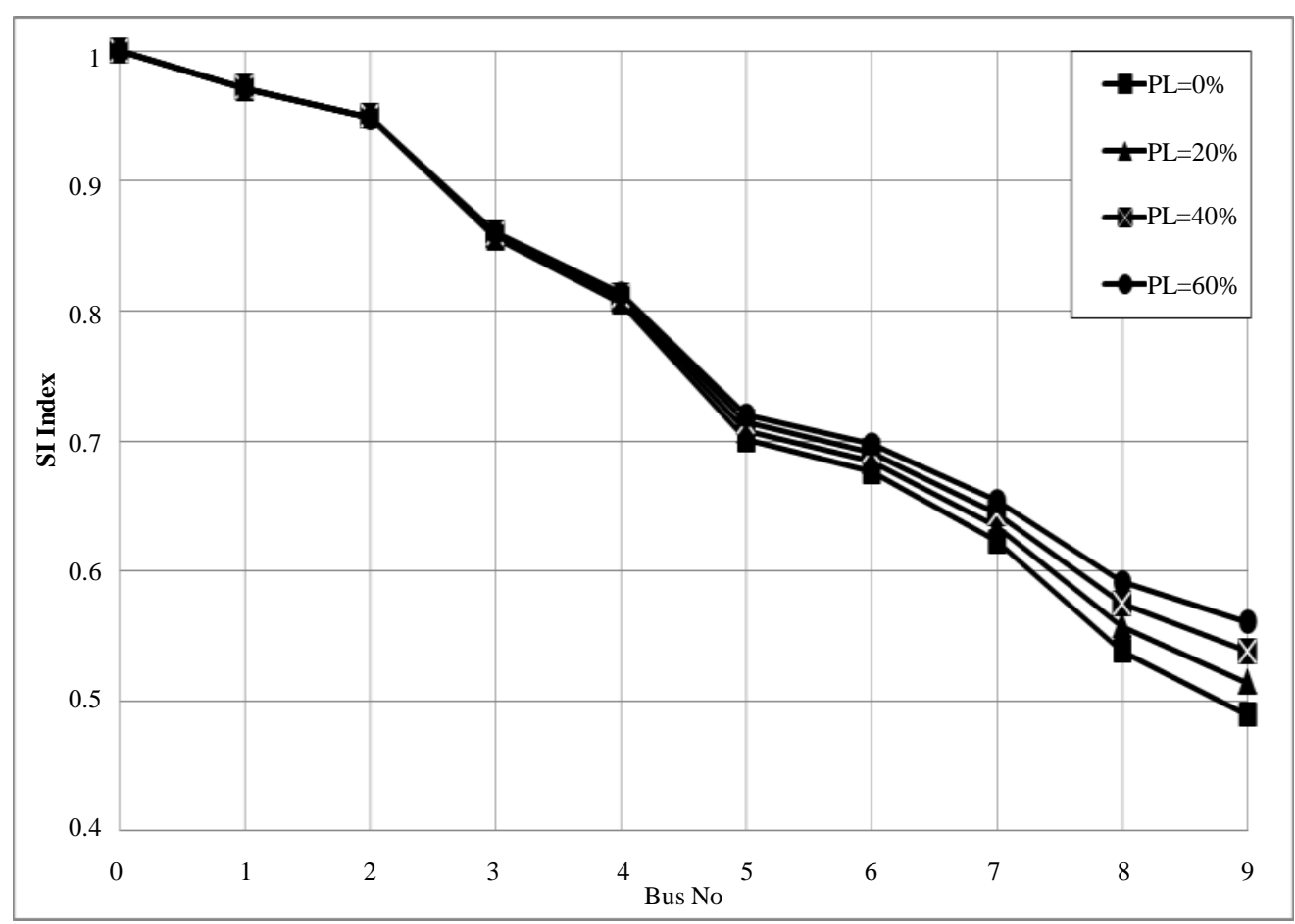

Figure 7. SI index at different PLs (0.9 lag p.f.).

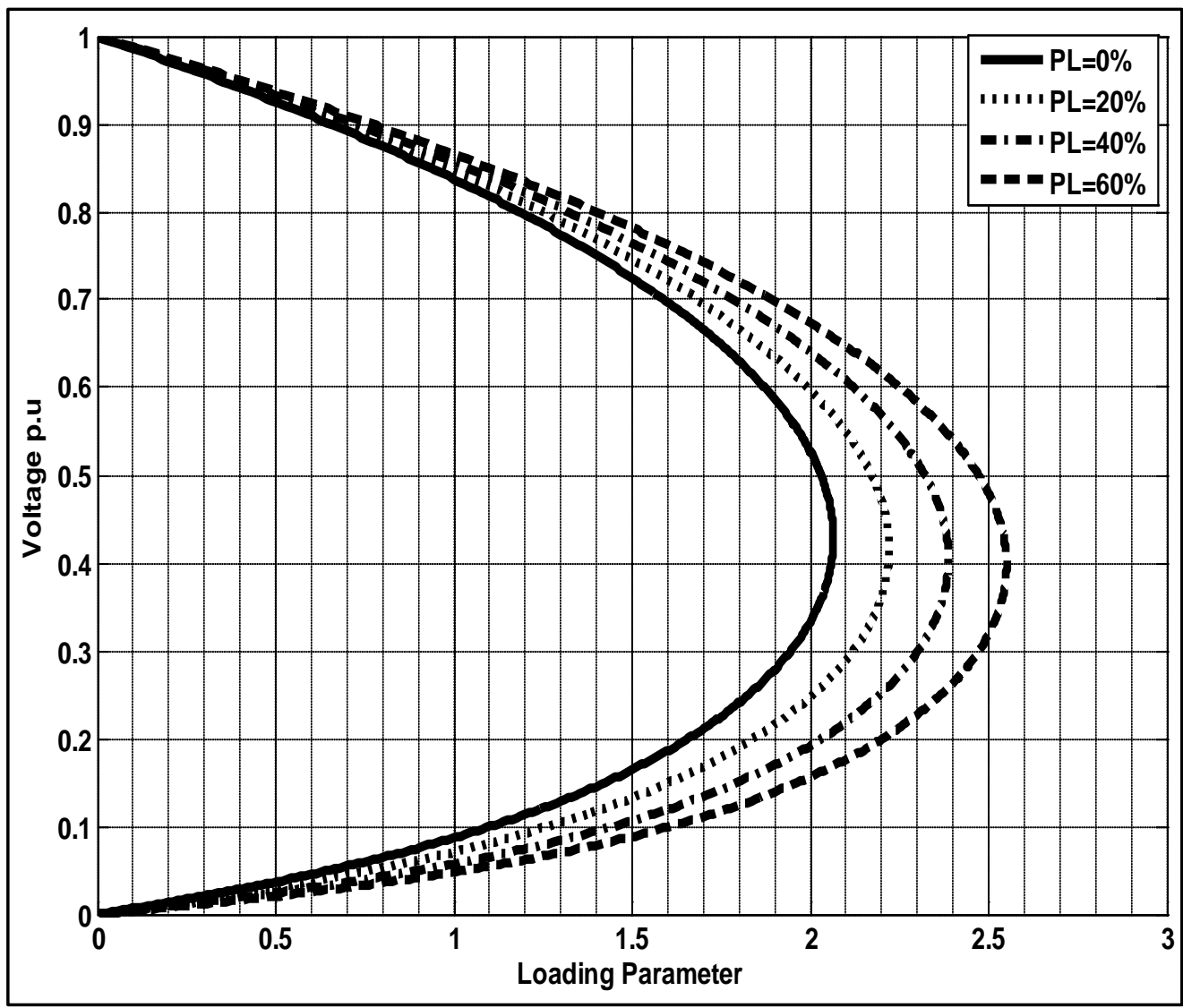

Figure $8 . \lambda-V$ curves at bus-9 with different PLs (lag p.f.). 
Results show that the impact of WT also depends on its power factor i.e. (in case of lead p.f enhance voltage stability and PLR more than lag p.f case).

WT installed at bus 9 with different PLs listed in following Table 1 for both 0.9 lead and lag p.f.

The Figures 3-10 show the impact of connection WT with different sizes and locations on voltage profile, SI index, $\lambda-V$ curves, active losses, and reactive losses; while Figure 11 shows the relation between penetration level and power loss of the system.

Tables 2-8 give the numerical values of the impact of connection WT with different sizes and locations on the system. While Table 9 and Table 10 gives the optimal size and location to install WT in the system.

Optimal penetration level at bus 9 to minimize the total system losses is shown in Figure 11.

\section{Second Case Study}

Another 33-bus radial distribution test system has been used [11] [19]. This test system has a main feeder and four laterals (sub-feeders). The data of the feeder is presented in Table C-2 in appendix C, the single line diagram shown in Figure 12. The system rated voltage is $11 \mathrm{kV}$.

Study the voltage stability enhancement and PLR by installing WT at bus 5 of distribution feeder with different PL\% listed in Table 11.

The Figures 13-20 show the impact of connection WT with different sizes and locations on voltage profile, SI index, $\lambda-V$ curves, active losses, and reactive losses; while Figure 21 shows the relation between penetration level and power loss of the system.

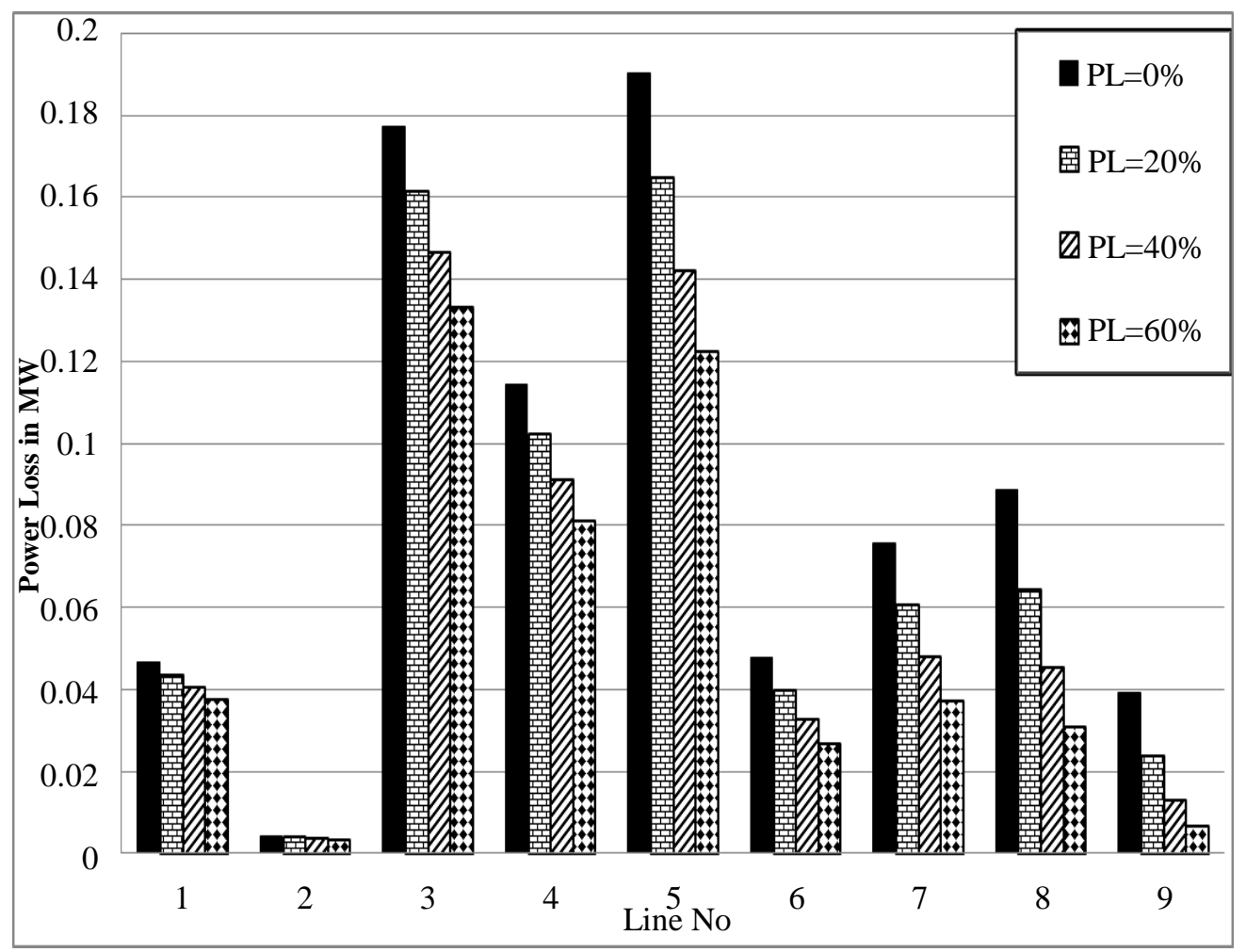

Figure 9. Power losses of 9-bus feeder at different PLs (lead p.f.).

Table 1. Different PLs of WT.

\begin{tabular}{ccccc}
\hline Case & 1 & 2 & 3 & 4 \\
\hline$P L \%$ & $0 \%$ & $20 \%$ & $40 \%$ & $60 \%$ \\
$P^{W T}(\mathrm{~kW})$ & 0.00 & 328 & 656 & 984 \\
\hline
\end{tabular}




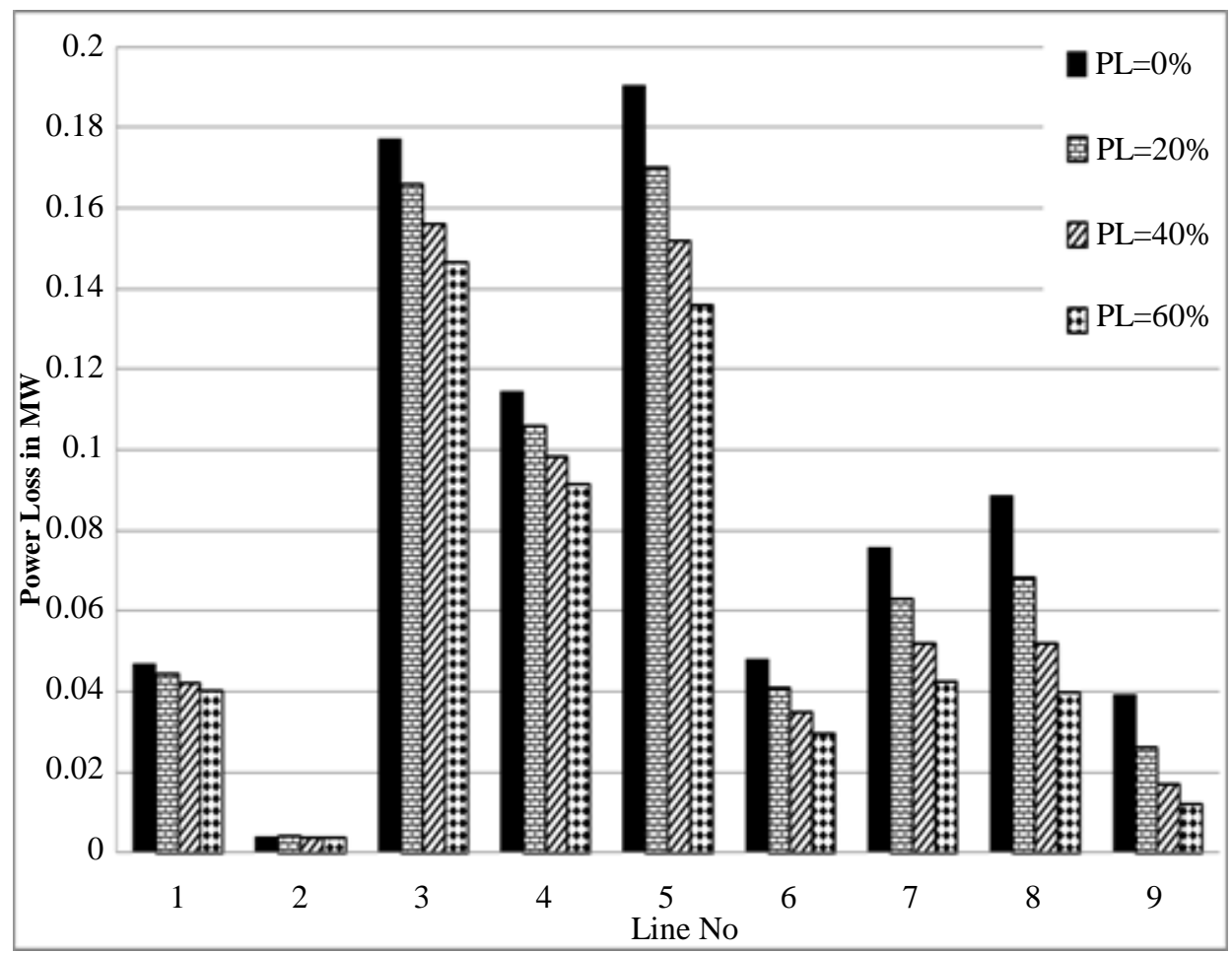

Figure 10. Power losses of 9-bus feeder at different PLs (lag p.f.).

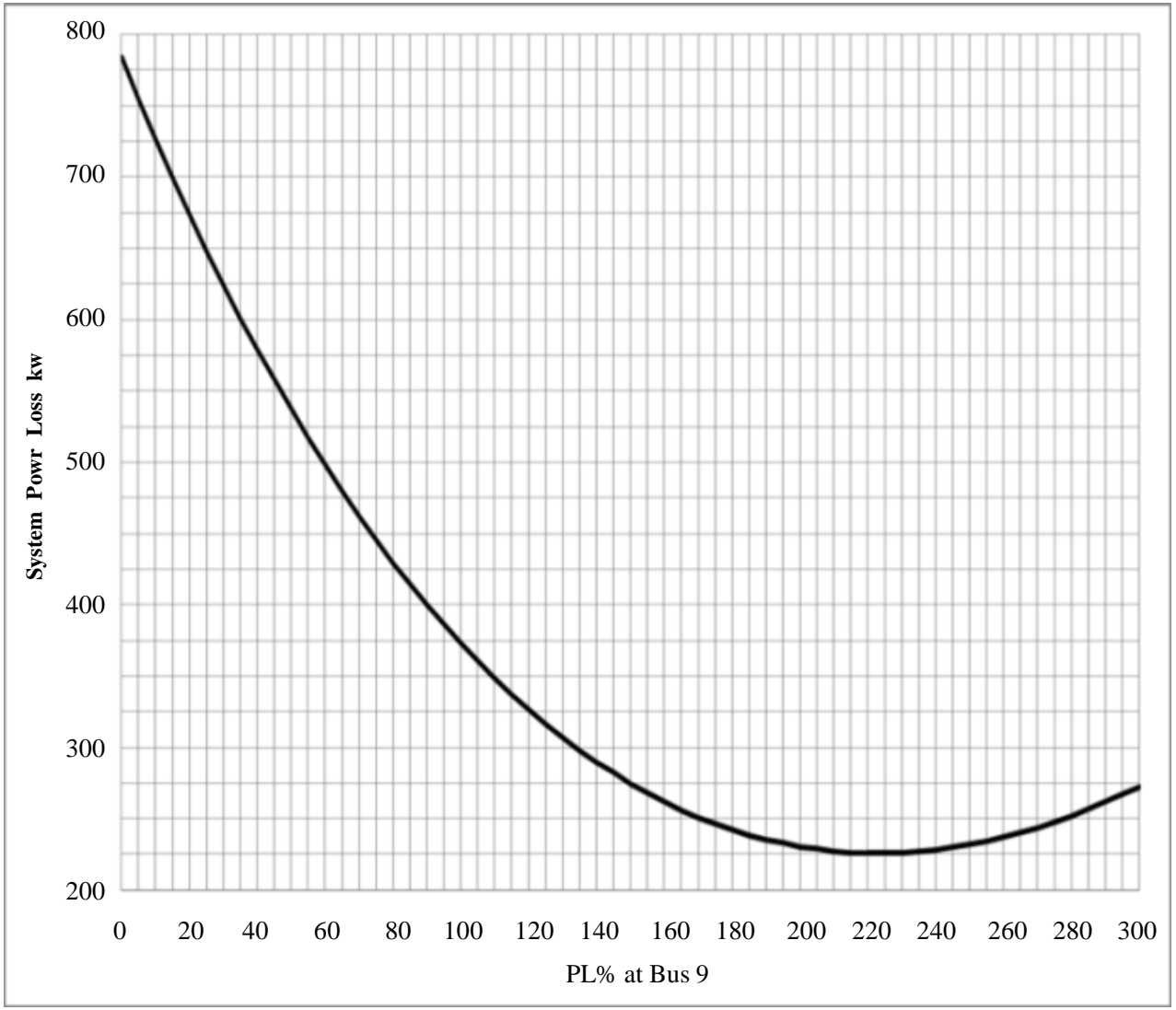

Figure 11. Total system real power loss at different PLs. 
Table 2. Bus Voltages at different PLs (0.9 lead p.f.).

\begin{tabular}{ccccc}
\hline Bus No & \multicolumn{3}{c}{ Bus Voltage in $\mathbf{p . u}$} \\
\cline { 2 - 4 } & $\boldsymbol{P L}=\mathbf{0} \%$ & $\boldsymbol{P L}=\mathbf{2 0} \%$ & $\boldsymbol{P L}=\mathbf{4 0} \%$ & $\boldsymbol{P L}=\mathbf{6 0} \%$ \\
$\mathbf{1}$ & 1.00000 & 1.00000 & 1.00000 & 1.00000 \\
$\mathbf{2}$ & 0.99290 & 0.99323 & 0.99353 & 0.99382 \\
$\mathbf{3}$ & 0.98737 & 0.98801 & 0.98862 & 0.98920 \\
$\mathbf{4}$ & 0.96340 & 0.96527 & 0.96705 & 0.96874 \\
$\mathbf{5}$ & 0.94801 & 0.95077 & 0.95339 & 0.95589 \\
$\mathbf{6}$ & 0.91716 & 0.92240 & 0.92738 & 0.93213 \\
$\mathbf{7}$ & 0.90716 & 0.91350 & 0.91952 & 0.92527 \\
$\mathbf{8}$ & 0.88895 & 0.89750 & 0.90563 & 0.91340 \\
$\mathbf{9}$ & 0.85869 & 0.87227 & 0.88520 & 0.89756 \\
\hline
\end{tabular}

Table 3. Bus voltages at different PLs (0.9 lag p.f.).

\begin{tabular}{ccccc}
\hline \multirow{2}{*}{ Bus No } & \multicolumn{3}{c}{ Bus Voltage in $\mathbf{p . u}$} \\
\cline { 2 - 4 } $\mathbf{0}$ & $\mathbf{P L}=\mathbf{0}$ & $\mathbf{P L}=\mathbf{2 0 \%}$ & $\mathbf{P L}=\mathbf{4 0} \%$ & $\boldsymbol{P L}=\mathbf{6 0} \%$ \\
\hline $\mathbf{1}$ & 1.00000 & 1.00000 & 1.00000 & 1.00000 \\
$\mathbf{2}$ & 0.99290 & 0.99295 & 0.99298 & 0.99300 \\
$\mathbf{3}$ & 0.98737 & 0.98734 & 0.98728 & 0.98719 \\
$\mathbf{4}$ & 0.96340 & 0.96376 & 0.96404 & 0.96425 \\
$\mathbf{5}$ & 0.94801 & 0.94881 & 0.94951 & 0.95010 \\
$\mathbf{6}$ & 0.91716 & 0.91917 & 0.92099 & 0.92263 \\
$\mathbf{7}$ & 0.90716 & 0.90969 & 0.91200 & 0.91409 \\
$\mathbf{8}$ & 0.88895 & 0.89280 & 0.89636 & 0.89964 \\
$\mathbf{9}$ & 0.85869 & 0.86550 & 0.87188 & 0.87786 \\
\hline
\end{tabular}

Table 4. SI index at different PLs (0.9 lead p.f.).

\begin{tabular}{|c|c|c|c|c|}
\hline \multirow{2}{*}{ Bus No } & \multicolumn{4}{|c|}{ SI Index } \\
\hline & $P L=0 \%$ & $P L=20 \%$ & $P L=40 \%$ & $P L=60 \%$ \\
\hline 0 & 1.00000 & 1.00000 & 1.00000 & 1.00000 \\
\hline 1 & 0.97112 & 0.97244 & 0.97368 & 0.97487 \\
\hline 2 & 0.94928 & 0.95185 & 0.95428 & 0.95658 \\
\hline 3 & 0.85618 & 0.86336 & 0.87019 & 0.87671 \\
\hline 4 & 0.80623 & 0.81584 & 0.82503 & 0.83385 \\
\hline 5 & 0.70087 & 0.71804 & 0.73456 & 0.75052 \\
\hline 6 & 0.67651 & 0.69575 & 0.71440 & 0.73254 \\
\hline 7 & 0.62256 & 0.64729 & 0.67143 & 0.69507 \\
\hline 8 & 0.53872 & 0.57522 & 0.61135 & 0.64720 \\
\hline 9 & 0.48966 & 0.53668 & 0.58422 & 0.63232 \\
\hline
\end{tabular}


Table 5. SI index at different PLs (0.9 lag p.f.).

\begin{tabular}{ccccc}
\hline \multirow{2}{*}{ Bus $\mathbf{N o}$} & \multicolumn{3}{c}{ SI Index } \\
\cline { 2 - 4 } $\mathbf{0}$ & $\mathbf{P L}=\mathbf{0} \%$ & $\mathbf{P L}=\mathbf{2 0} \%$ & $\mathbf{P L}=\mathbf{4 0} \%$ & $\boldsymbol{P L}=\mathbf{6 0} \%$ \\
$\mathbf{1}$ & 1.00000 & 1.00000 & 1.00000 & 1.00000 \\
$\mathbf{2}$ & 0.97112 & 0.97134 & 0.97150 & 0.97161 \\
$\mathbf{3}$ & 0.94928 & 0.94922 & 0.94905 & 0.94877 \\
$\mathbf{4}$ & 0.85618 & 0.85780 & 0.85911 & 0.86015 \\
$\mathbf{5}$ & 0.80623 & 0.80908 & 0.81155 & 0.81367 \\
$\mathbf{6}$ & 0.70087 & 0.70781 & 0.71410 & 0.71979 \\
$\mathbf{7}$ & 0.67651 & 0.68421 & 0.69126 & 0.69770 \\
$\mathbf{8}$ & 0.62256 & 0.63378 & 0.64423 & 0.65396 \\
$\mathbf{9}$ & 0.53872 & 0.55727 & 0.57489 & 0.59160 \\
\hline
\end{tabular}

Table 6. Power losses of 9-bus feeder at different PLs (lead p.f.).

\begin{tabular}{ccccc}
\hline Line No & \multicolumn{2}{c}{ Power Loss in $\mathbf{M W}$} \\
\cline { 2 - 4 } & $\boldsymbol{P L}=\mathbf{0} \%$ & $\boldsymbol{P L}=\mathbf{2 0} \%$ & $\boldsymbol{P L}=\mathbf{4 0} \%$ & $\boldsymbol{P L}=\mathbf{6 0} \%$ \\
\hline 1 & 0.04666 & 0.04328 & 0.04019 & 0.03734 \\
3 & 0.00406 & 0.00372 & 0.00341 & 0.00313 \\
4 & 0.17725 & 0.16113 & 0.14650 & 0.13316 \\
5 & 0.11438 & 0.10197 & 0.09081 & 0.08075 \\
6 & 0.19024 & 0.16464 & 0.14209 & 0.12218 \\
7 & 0.04777 & 0.03954 & 0.03249 & 0.02649 \\
8 & 0.07574 & 0.06048 & 0.04772 & 0.03713 \\
9 & 0.08847 & 0.06430 & 0.04522 & 0.03058 \\
Total $\boldsymbol{M W}$ Loss & 0.03931 & 0.02373 & 0.01297 & 0.00644 \\
\end{tabular}

Table 7. Power losses of 9-bus feeder at different PLs (lag p.f.).

\begin{tabular}{ccccc}
\hline \multirow{2}{*}{ Line No } & \multicolumn{2}{c}{ Power Losses in $\boldsymbol{M W}$} \\
\cline { 2 - 4 } & $\mathbf{P L}=\mathbf{0} \%$ & $\mathbf{P L}=\mathbf{2 0} \%$ & $\boldsymbol{P L}=\mathbf{4 0 \%}$ & $\boldsymbol{P L}=\mathbf{6 0} \%$ \\
\hline 2 & 0.04666 & 0.04328 & 0.04019 & 0.03734 \\
3 & 0.00406 & 0.00372 & 0.00341 & 0.00313 \\
4 & 0.17725 & 0.16113 & 0.14650 & 0.13316 \\
5 & 0.11438 & 0.10197 & 0.09081 & 0.08075 \\
6 & 0.19024 & 0.16464 & 0.14209 & 0.12218 \\
7 & 0.04777 & 0.03954 & 0.03249 & 0.02649 \\
8 & 0.07574 & 0.06048 & 0.04772 & 0.03713 \\
9 & 0.08847 & 0.06430 & 0.04522 & 0.03058 \\
Total $\boldsymbol{M W}$ Loss & 0.03931 & 0.02373 & 0.01297 & 0.00644 \\
\hline
\end{tabular}


Table 8. Voltage and voltage index of bus-9 in all cases.

\begin{tabular}{|c|c|c|c|c|c|}
\hline \multicolumn{2}{|c|}{ Case } & 1 & 2 & 3 & 4 \\
\hline \multicolumn{2}{|c|}{$P L \%$} & $0 \%$ & $20 \%$ & $40 \%$ & $60 \%$ \\
\hline \multicolumn{2}{|c|}{$P^{W T}(\mathrm{~kW})$} & 0.0000 & 328.00 & 656.00 & 984.00 \\
\hline \multirow{5}{*}{0.9 Lead p.f } & $V_{\text {Bus-9 }} p . u$ & 0.83750 & 0.85648 & 0.87457 & 0.89188 \\
\hline & $S I_{\text {Bus-9 }}$ & 0.48966 & 0.53668 & 0.58422 & 0.63232 \\
\hline & $\lambda_{\max }$ & 2.0645 & 2.3026 & 2.5836 & 2.9045 \\
\hline & $P_{\text {Loss }}(\mathrm{kW})$ & 783.88 & 662.79 & 561.40 & 477.20 \\
\hline & $P L R \%$ & $0.00 \%$ & $15.45 \%$ & $28.38 \%$ & $39.12 \%$ \\
\hline \multirow{5}{*}{0.9 Lag. p.f } & $V_{\text {Bus-9 }} p \cdot u$ & 0.83750 & 0.84742 & 0.85682 & 0.86573 \\
\hline & $S I_{\text {Bus-9 }}$ & 0.48966 & 0.51416 & 0.53793 & 0.56100 \\
\hline & $\lambda_{\max }$ & 2.0645 & 2.2216 & 2.3874 & 2.5505 \\
\hline & $P_{\text {Loss }}(\mathrm{kW})$ & 783.88 & 687.27 & 606.75 & 541.14 \\
\hline & $P L R \%$ & $0.00 \%$ & $12.32 \%$ & $22.60 \%$ & $30.96 \%$ \\
\hline
\end{tabular}

Table 9. Optimal size of $W T$ at various buses.

\begin{tabular}{ccc}
\hline Bus No & Optimal Size of WT (MW) & Line Losses $(\mathbf{k W})$ \\
\hline $\mathbf{0}$ & 13.15 & 783.9 \\
$\mathbf{1}$ & 13.60 & 737.0 \\
$\mathbf{2}$ & 13.60 & 732.2 \\
$\mathbf{3}$ & 11.13 & 556.4 \\
$\mathbf{4}$ & 9.890 & 452.4 \\
$\mathbf{5}$ & 7.920 & 279.3 \\
$\mathbf{6}$ & 7.170 & 246.1 \\
$\mathbf{7}$ & 6.060 & 204.7 \\
$\mathbf{8}$ & 4.580 & 192.2 \\
$\mathbf{9}$ & 3.710 & 226.0 \\
\hline
\end{tabular}

Table 10. Optimal location for installing WT on system.

\begin{tabular}{|c|c|}
\hline WT Optimal Location & Bus 8 \\
\hline WT optimal Size & 4.57 MW \\
\hline Active Power loss without $W T$ & $783.870 \mathrm{~kW}$ \\
\hline Reactive Power loss without $W T$ & $1036.50 \mathrm{kVAr}$ \\
\hline Active Power loss with $W T$ & $192.16 \mathrm{~kW}$ \\
\hline Reactive Power loss with $W T$ & $296.49 \mathrm{kVAr}$ \\
\hline Minimum Voltage without $W T$ & 0.83750 p.u (Bus 9) \\
\hline Maximum Voltage without $W T$ & 1.00000 p.u (Bus 0) \\
\hline Minimum Voltage with $W T$ & 0.95459 p.u (Bus 9) \\
\hline Maximum Voltage with $W T$ & 1.00000 p.u (Bus 0) \\
\hline
\end{tabular}


Table 11. Different PLs of WT.

\begin{tabular}{ccccc}
\hline Case & $\mathbf{1}$ & $\mathbf{2}$ & $\mathbf{3}$ & $\mathbf{4}$ \\
\hline $\boldsymbol{P L \%}$ & $0 \%$ & $20 \%$ & $40 \%$ & $60 \%$ \\
$\boldsymbol{P}^{W T}(\mathbf{k W})$ & 0.00 & 258.68 & 517.35 & 776.03 \\
\hline
\end{tabular}

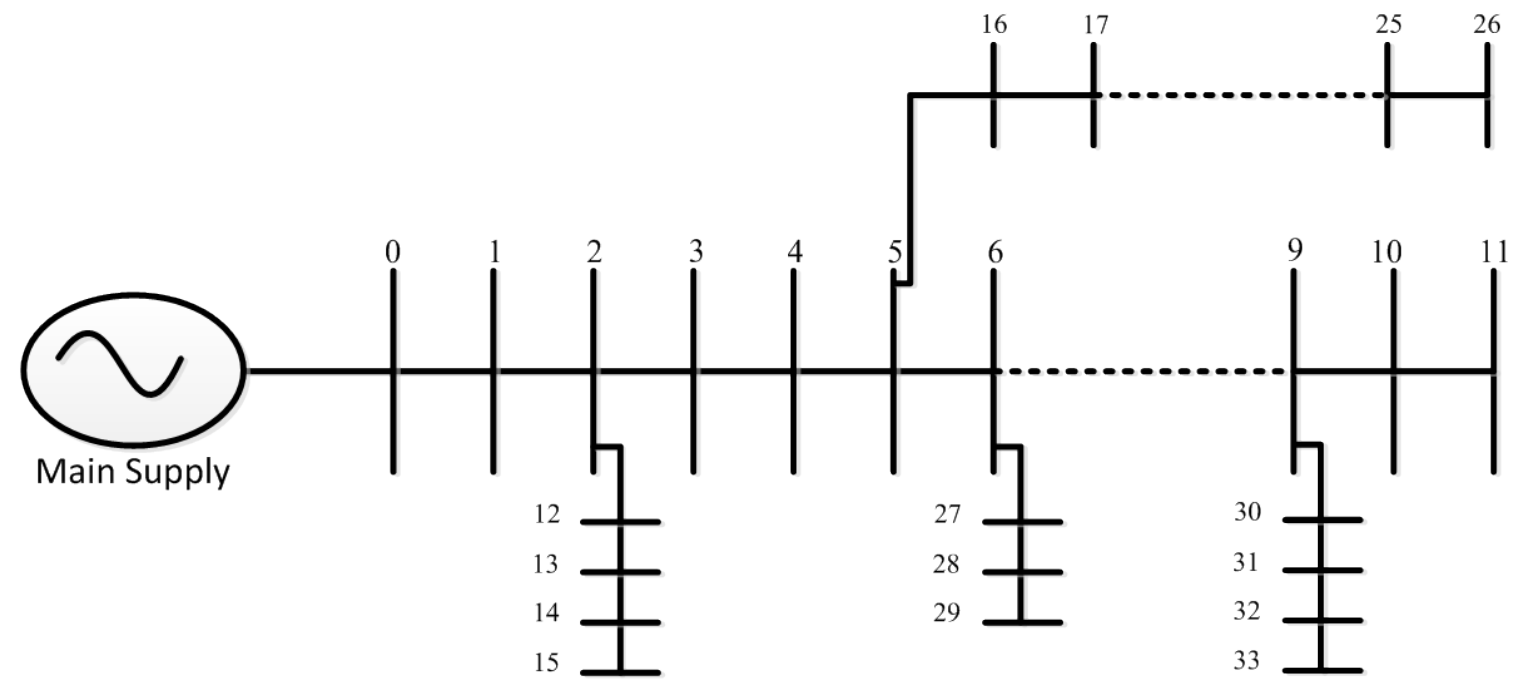

Figure 12. Single line diagram of feeder 33-bus laterals and sub-lateral.

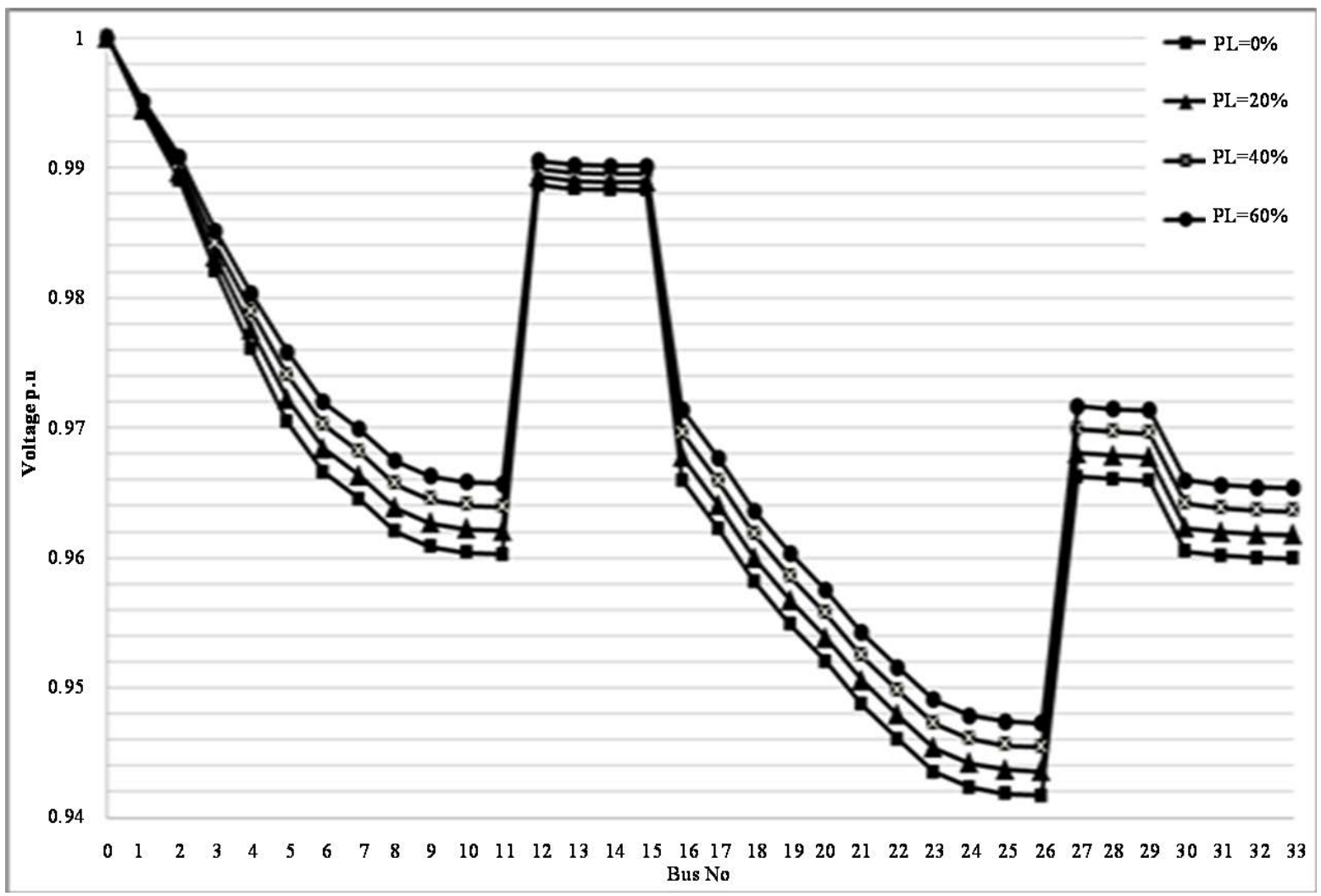

Figure 13. Bus voltages at different PLs (0.9 lead p.f.). 


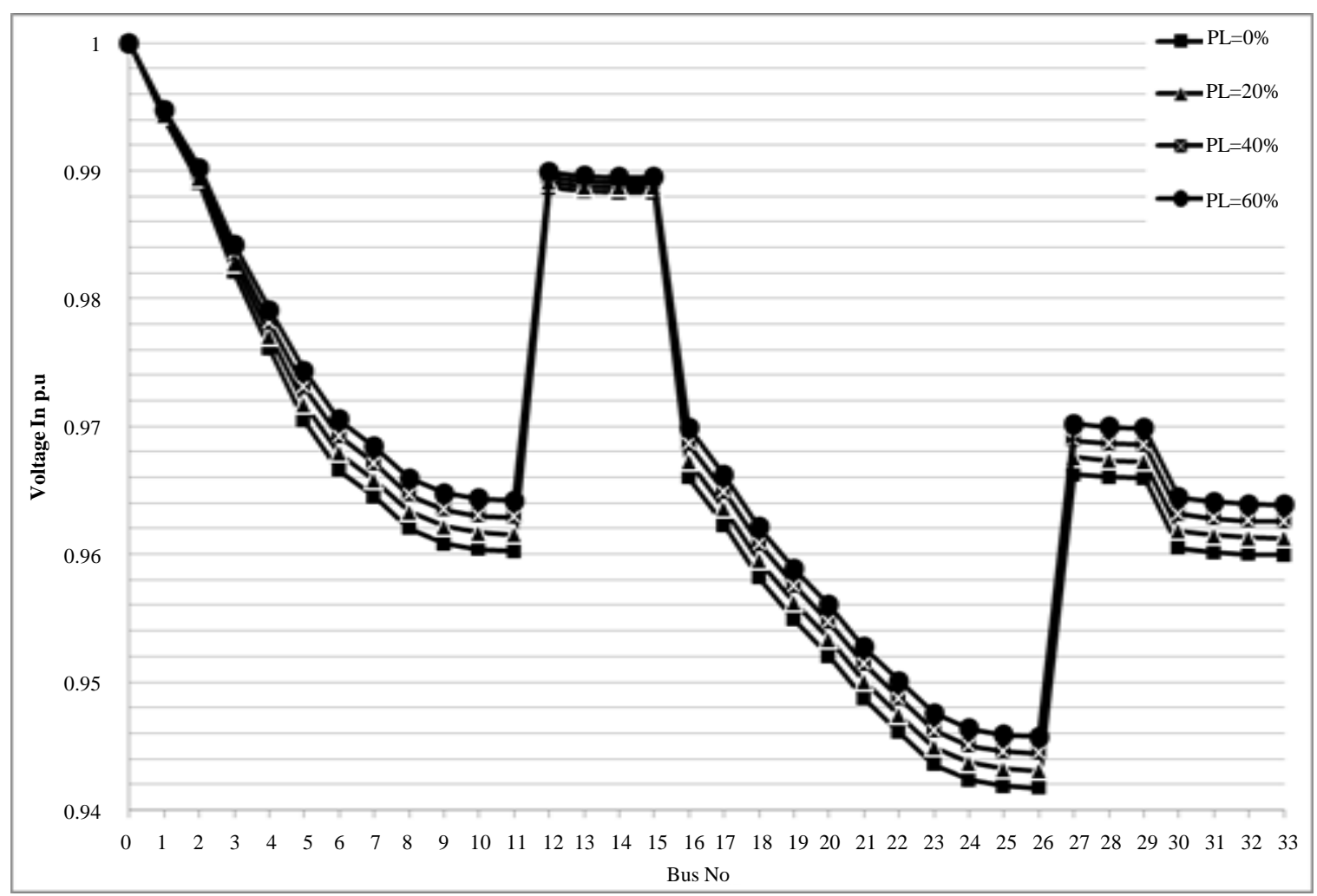

Figure 14. Bus voltages at different PLs (0.9 lag p.f.).

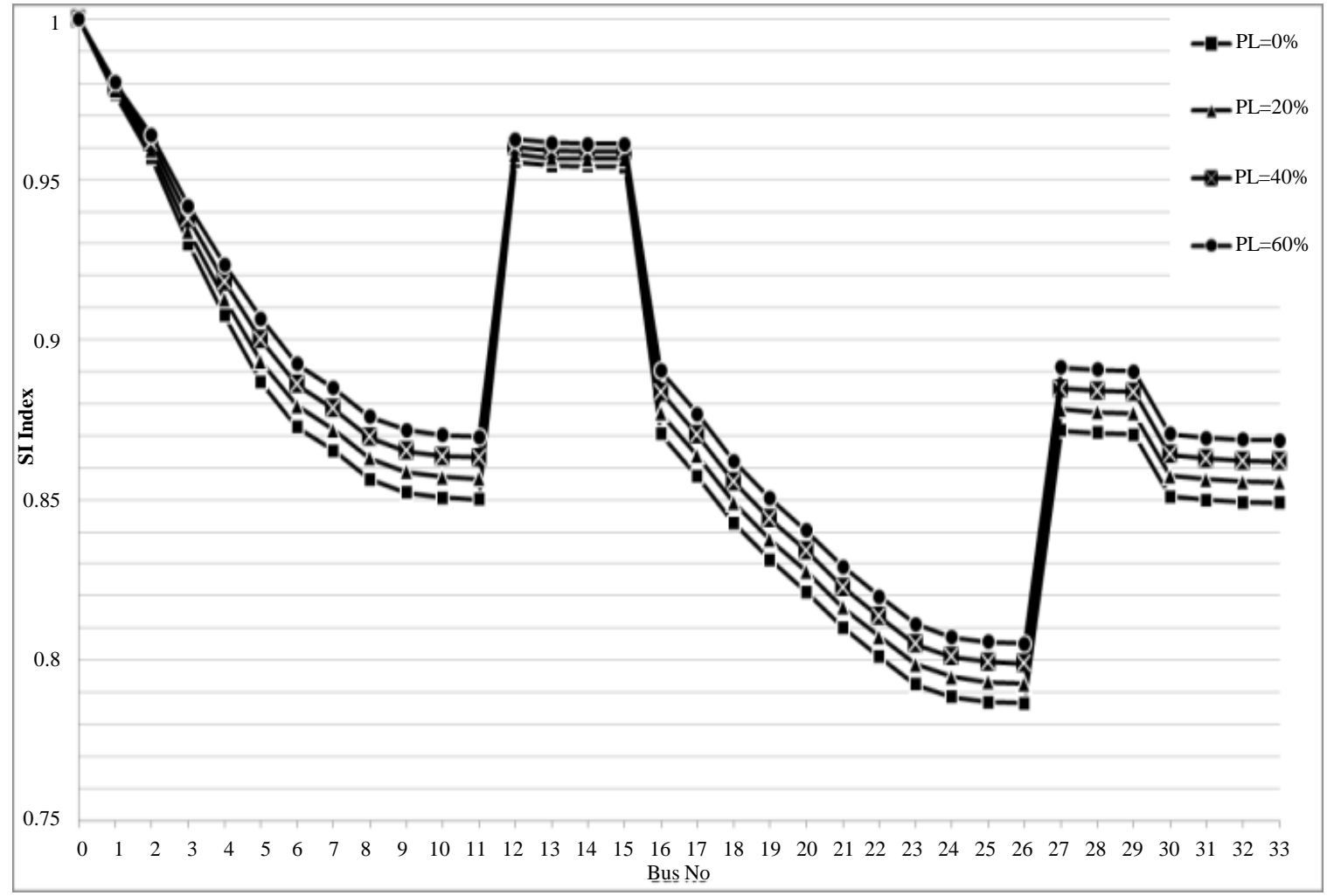

Figure 15. SI index at different PLs (0.9 lead p.f.). 


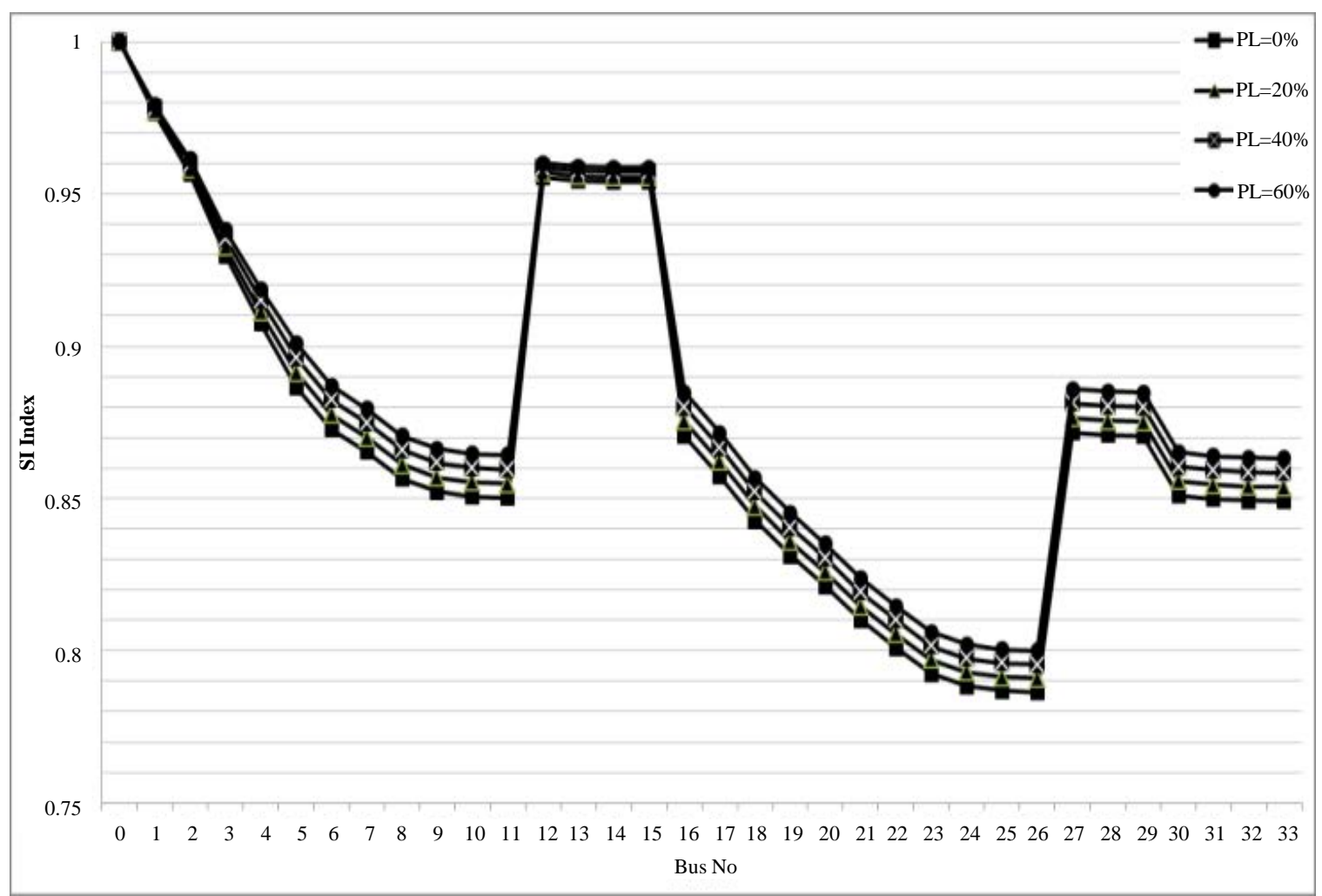

Figure 16. SI index at different PLs (0.9 lag p.f.).

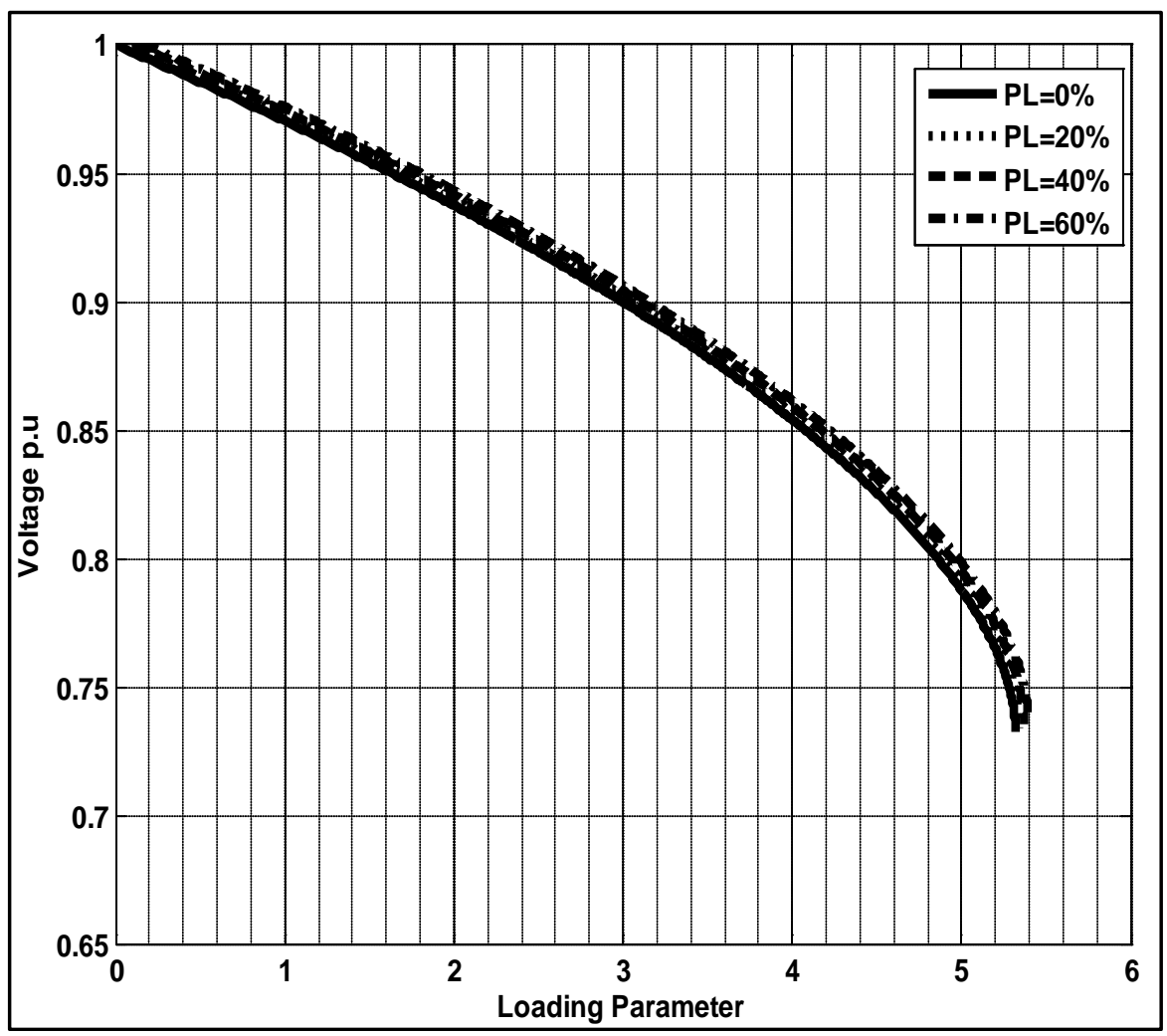

Figure 17. $\lambda-V$ curves at bus-5 with different PLs (lead p.f.). 


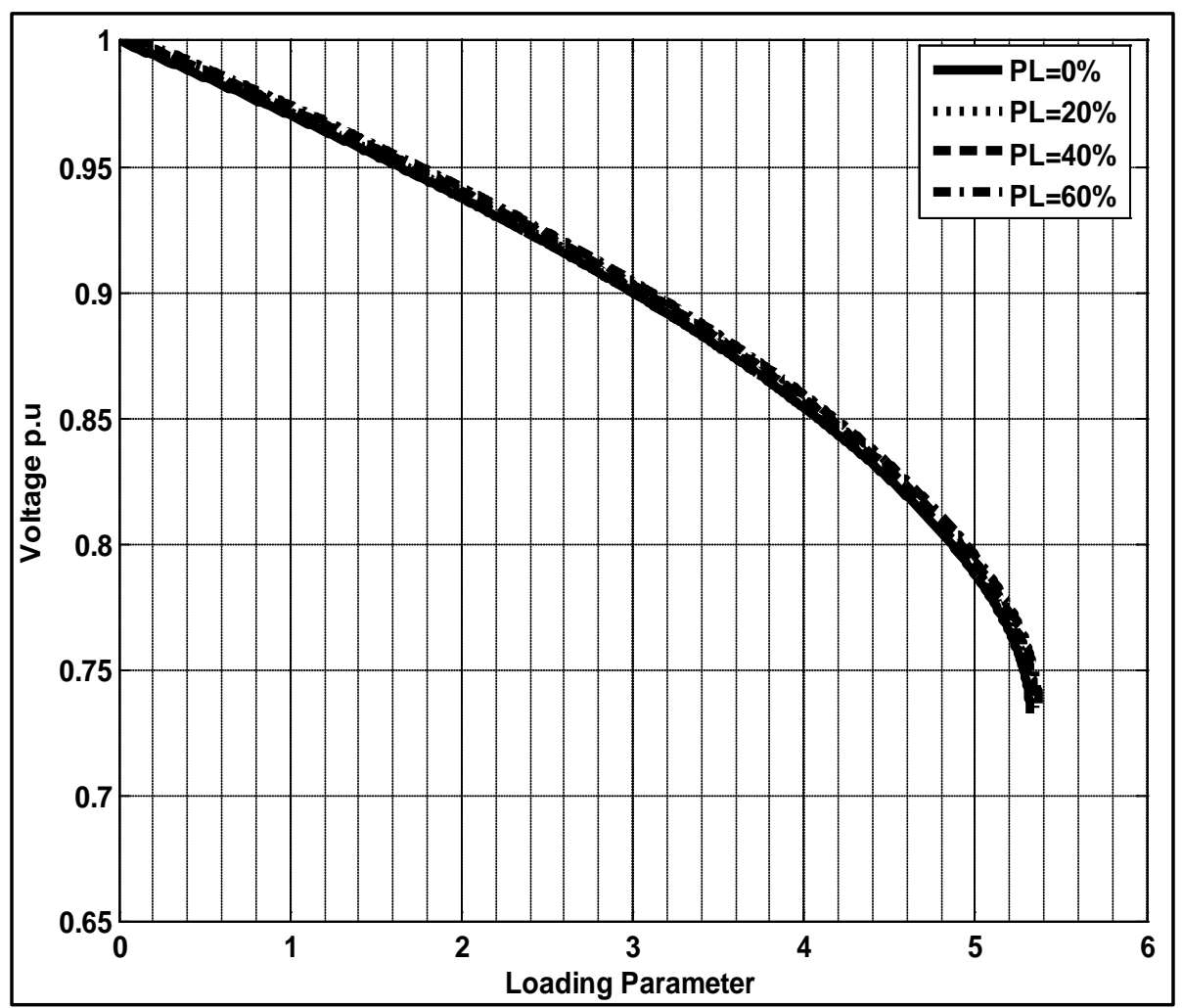

Figure 18. $\lambda-V$ curves at bus-5 with different PLs (lag p.f.).

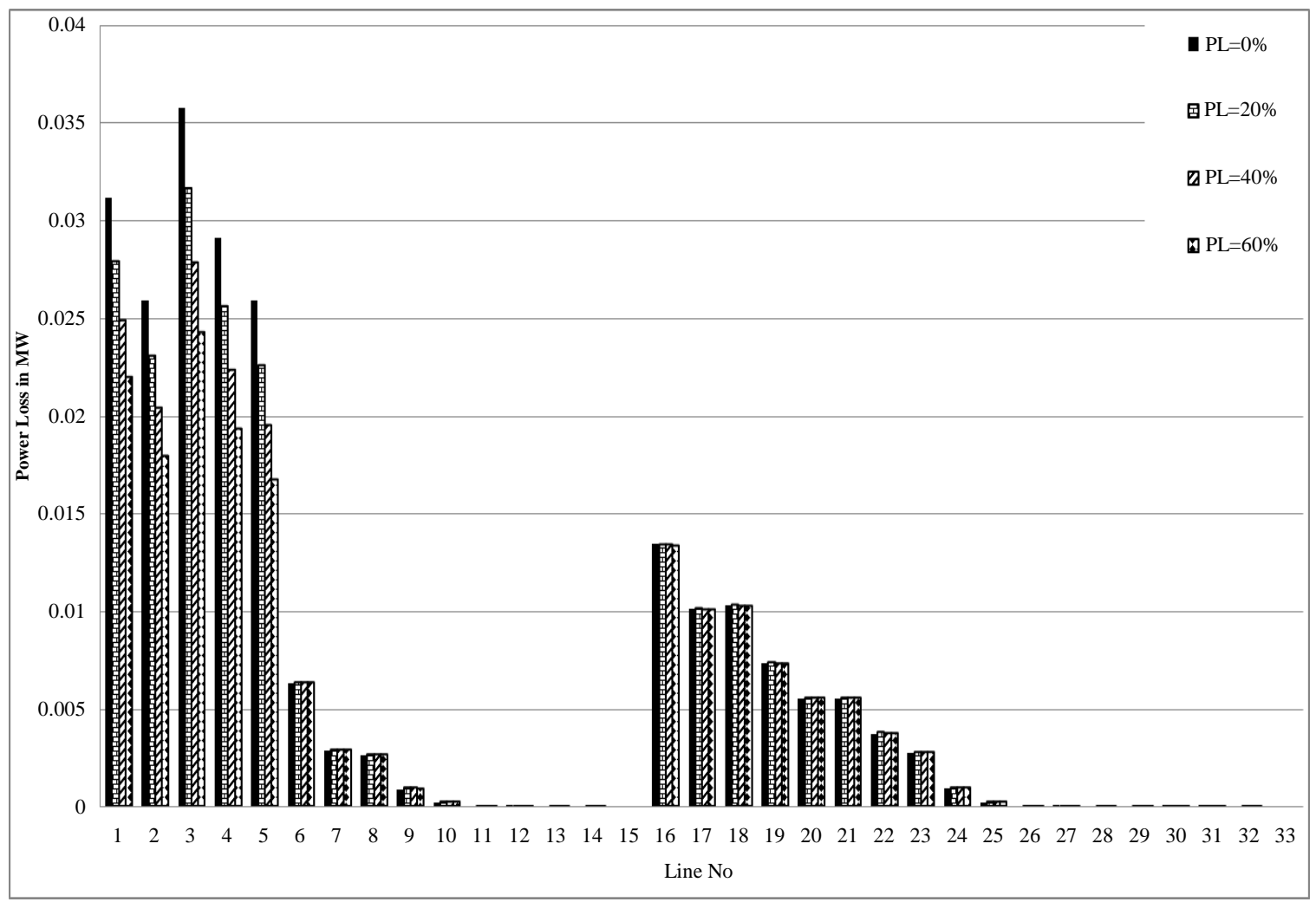

Figure 19. Power loss of 33-bus at different PLs (lead p.f.). 


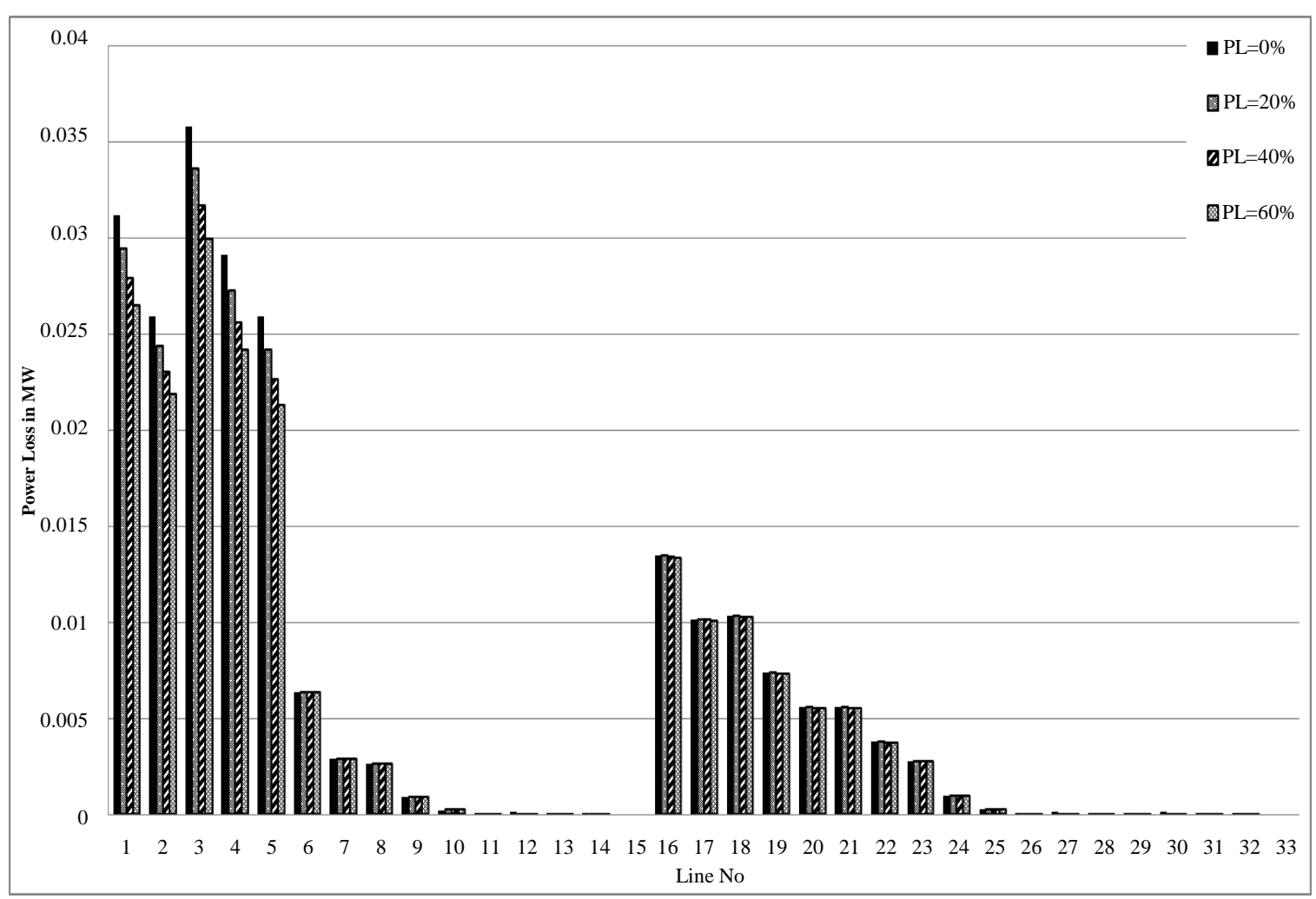

Figure 20. Power loss of 33-bus at different PLs (lag p.f.).

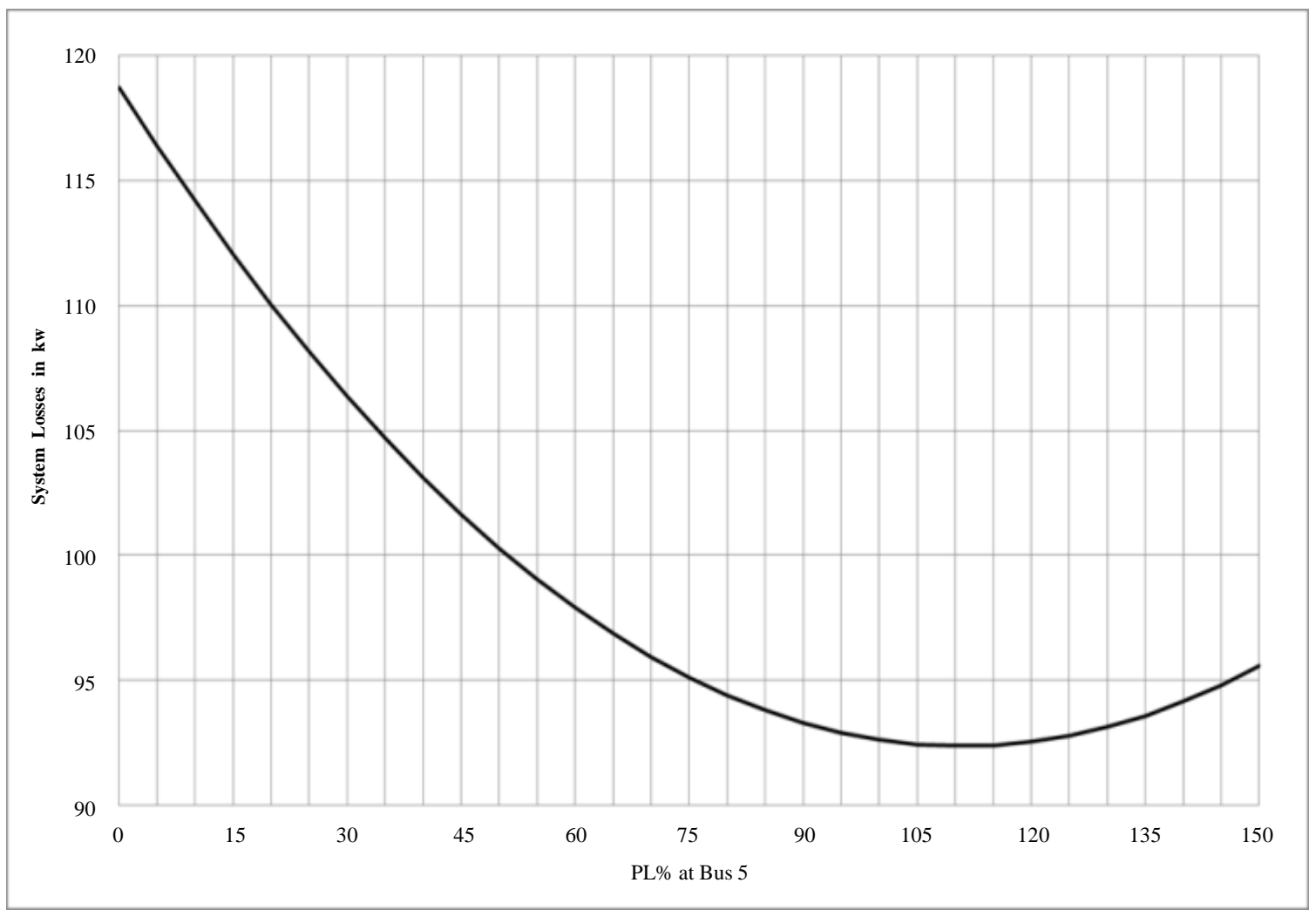

Figure 21. Total system real power losses at different PLs. 
Tables 12-18 give the numerical values of the impact of connection WT with different sizes and locations on the system, while Table 19 and Table 20 give the optimal size and location to install WT in the system.

Optimal penetration level at bus 5 to minimize the total system losses is $110 \%$ as shown in Figure 21 .

Table 12. Bus voltages at different PLs (0.9 lead p.f.).

\begin{tabular}{|c|c|c|c|c|}
\hline \multirow{2}{*}{ Bus No } & \multicolumn{4}{|c|}{ Bus Voltage in p.u } \\
\hline & $P L=0 \%$ & $P L=20 \%$ & $P L=40 \%$ & $P L=60 \%$ \\
\hline $\mathbf{0}$ & 1.00000 & 1.00000 & 1.0000 & 1.00000 \\
\hline 1 & 0.99414 & 0.99446 & 0.99477 & 0.99509 \\
\hline 2 & 0.98902 & 0.98963 & 0.99024 & 0.99085 \\
\hline 3 & 0.98206 & 0.98309 & 0.98412 & 0.98515 \\
\hline 4 & 0.97606 & 0.97748 & 0.97890 & 0.98031 \\
\hline 5 & 0.97042 & 0.97222 & 0.97402 & 0.97581 \\
\hline 6 & 0.96659 & 0.96840 & 0.97020 & 0.97200 \\
\hline 7 & 0.96449 & 0.96630 & 0.96811 & 0.96991 \\
\hline 8 & 0.96202 & 0.96384 & 0.96565 & 0.96745 \\
\hline 9 & 0.96083 & 0.96265 & 0.96447 & 0.96627 \\
\hline 10 & 0.96038 & 0.96220 & 0.96401 & 0.96582 \\
\hline 11 & 0.96024 & 0.96206 & 0.96388 & 0.96568 \\
\hline 12 & 0.98869 & 0.98930 & 0.98991 & 0.99051 \\
\hline 13 & 0.98838 & 0.98899 & 0.98960 & 0.99021 \\
\hline 14 & 0.98830 & 0.98891 & 0.98952 & 0.99013 \\
\hline 15 & 0.98829 & 0.98890 & 0.98951 & 0.99012 \\
\hline 16 & 0.96597 & 0.96778 & 0.96958 & 0.97138 \\
\hline 17 & 0.96226 & 0.96408 & 0.96589 & 0.96770 \\
\hline 18 & 0.95817 & 0.95999 & 0.96181 & 0.96362 \\
\hline 19 & 0.95487 & 0.95671 & 0.95853 & 0.96035 \\
\hline 20 & 0.95201 & 0.95385 & 0.95568 & 0.95750 \\
\hline 21 & 0.94874 & 0.95059 & 0.95242 & 0.95426 \\
\hline 22 & 0.94606 & 0.94791 & 0.94975 & 0.95158 \\
\hline 23 & 0.94353 & 0.94539 & 0.94724 & 0.94908 \\
\hline 24 & 0.94232 & 0.94418 & 0.94603 & 0.94787 \\
\hline 25 & 0.94185 & 0.94371 & 0.94556 & 0.94740 \\
\hline 26 & 0.94171 & 0.94357 & 0.94542 & 0.94727 \\
\hline 27 & 0.96625 & 0.96806 & 0.96987 & 0.97167 \\
\hline 28 & 0.96603 & 0.96784 & 0.96965 & 0.97144 \\
\hline 29 & 0.96592 & 0.96773 & 0.96953 & 0.97133 \\
\hline 30 & 0.96049 & 0.96231 & 0.96413 & 0.96594 \\
\hline 31 & 0.96015 & 0.96197 & 0.96379 & 0.96560 \\
\hline 32 & 0.95998 & 0.96180 & 0.96362 & 0.96543 \\
\hline 33 & 0.95992 & 0.96175 & 0.96356 & 0.96537 \\
\hline
\end{tabular}


Table 13. Bus voltages at different PLs (0.9 lag p.f.).

\begin{tabular}{|c|c|c|c|c|}
\hline \multirow{2}{*}{ Bus No } & \multicolumn{4}{|c|}{ Bus Voltage in p.u } \\
\hline & $P L=0 \%$ & $P L=20 \%$ & $P L=40 \%$ & $P L=60 \%$ \\
\hline $\mathbf{0}$ & 1.00000 & 1.00000 & 1.00000 & 1.00000 \\
\hline 1 & 0.99414 & 0.99435 & 0.99456 & 0.99477 \\
\hline 2 & 0.98902 & 0.98943 & 0.98983 & 0.99023 \\
\hline 3 & 0.98206 & 0.98278 & 0.98350 & 0.98422 \\
\hline 4 & 0.97606 & 0.97708 & 0.97809 & 0.97910 \\
\hline 5 & 0.97042 & 0.97173 & 0.97303 & 0.97433 \\
\hline 6 & 0.96659 & 0.96790 & 0.96921 & 0.97051 \\
\hline 7 & 0.96449 & 0.96580 & 0.96711 & 0.96842 \\
\hline 8 & 0.96202 & 0.96334 & 0.96465 & 0.96596 \\
\hline 9 & 0.96083 & 0.96215 & 0.96347 & 0.96478 \\
\hline 10 & 0.96038 & 0.96170 & 0.96301 & 0.96433 \\
\hline 11 & 0.96024 & 0.96156 & 0.96288 & 0.96419 \\
\hline 12 & 0.98869 & 0.98909 & 0.98950 & 0.98990 \\
\hline 13 & 0.98838 & 0.98879 & 0.98919 & 0.98959 \\
\hline 14 & 0.98830 & 0.98870 & 0.98911 & 0.98951 \\
\hline 15 & 0.98829 & 0.98870 & 0.98910 & 0.98950 \\
\hline 16 & 0.96597 & 0.96728 & 0.96859 & 0.96990 \\
\hline 17 & 0.96226 & 0.96358 & 0.96489 & 0.96620 \\
\hline 18 & 0.95817 & 0.95949 & 0.96081 & 0.96213 \\
\hline 19 & 0.95487 & 0.95620 & 0.95753 & 0.95885 \\
\hline 20 & 0.95201 & 0.95334 & 0.95467 & 0.95600 \\
\hline 21 & 0.94874 & 0.95008 & 0.95141 & 0.95274 \\
\hline 22 & 0.94606 & 0.94740 & 0.94874 & 0.95007 \\
\hline 23 & 0.94353 & 0.94488 & 0.94622 & 0.94755 \\
\hline 24 & 0.94232 & 0.94367 & 0.94501 & 0.94634 \\
\hline 25 & 0.94185 & 0.94320 & 0.94454 & 0.94588 \\
\hline 26 & 0.94171 & 0.94306 & 0.9444 & 0.94574 \\
\hline 27 & 0.96625 & 0.96757 & 0.96888 & 0.97018 \\
\hline 28 & 0.96603 & 0.96734 & 0.96865 & 0.96996 \\
\hline 29 & 0.96592 & 0.96723 & 0.96854 & 0.96985 \\
\hline 30 & 0.96049 & 0.96181 & 0.96313 & 0.96444 \\
\hline 31 & 0.96015 & 0.96147 & 0.96279 & 0.96410 \\
\hline 32 & 0.95998 & 0.96130 & 0.96262 & 0.96393 \\
\hline 33 & 0.95992 & 0.96125 & 0.96256 & 0.96388 \\
\hline
\end{tabular}


Table 14. SI index at different PLs (0.9 lead p.f.).

\begin{tabular}{|c|c|c|c|c|}
\hline \multirow{2}{*}{ Bus No } & \multicolumn{4}{|c|}{ SI index } \\
\hline & $P L=0 \%$ & $P L=20 \%$ & $P L=40 \%$ & $P L=60 \%$ \\
\hline $\mathbf{0}$ & 1.00000 & 1.00000 & 1.00000 & 1.00000 \\
\hline 1 & 0.97654 & 0.97782 & 0.97909 & 0.98035 \\
\hline 2 & 0.95664 & 0.95903 & 0.96140 & 0.96377 \\
\hline 3 & 0.92982 & 0.93378 & 0.93774 & 0.94169 \\
\hline 4 & 0.90741 & 0.91273 & 0.91804 & 0.92336 \\
\hline 5 & 0.88661 & 0.89324 & 0.89989 & 0.90654 \\
\hline 6 & 0.87280 & 0.87936 & 0.88593 & 0.89251 \\
\hline 7 & 0.86530 & 0.87183 & 0.87837 & 0.88492 \\
\hline 8 & 0.85646 & 0.86296 & 0.86947 & 0.87599 \\
\hline 9 & 0.85228 & 0.85876 & 0.86525 & 0.87175 \\
\hline 10 & 0.85066 & 0.85714 & 0.86362 & 0.87012 \\
\hline 11 & 0.85018 & 0.85665 & 0.86314 & 0.86963 \\
\hline 12 & 0.95551 & 0.95787 & 0.96023 & 0.96259 \\
\hline 13 & 0.95433 & 0.95669 & 0.95905 & 0.96140 \\
\hline 14 & 0.95401 & 0.95637 & 0.95873 & 0.96108 \\
\hline 15 & 0.95398 & 0.95635 & 0.95870 & 0.96106 \\
\hline 16 & 0.87053 & 0.87708 & 0.88365 & 0.89022 \\
\hline 17 & 0.85728 & 0.86378 & 0.87029 & 0.87682 \\
\hline 18 & 0.84276 & 0.84920 & 0.85566 & 0.86213 \\
\hline 19 & 0.83126 & 0.83767 & 0.84408 & 0.85051 \\
\hline 20 & 0.82135 & 0.82772 & 0.83410 & 0.84049 \\
\hline 21 & 0.81011 & 0.81644 & 0.82277 & 0.82912 \\
\hline 22 & 0.80100 & 0.80728 & 0.81358 & 0.81989 \\
\hline 23 & 0.79249 & 0.79874 & 0.80501 & 0.81128 \\
\hline 24 & 0.78845 & 0.79468 & 0.80093 & 0.80719 \\
\hline 25 & 0.78689 & 0.79312 & 0.79937 & 0.80562 \\
\hline 26 & 0.78643 & 0.79266 & 0.79890 & 0.80515 \\
\hline 27 & 0.87168 & 0.87824 & 0.88480 & 0.89138 \\
\hline 28 & 0.87088 & 0.87743 & 0.88399 & 0.89056 \\
\hline 29 & 0.87047 & 0.87702 & 0.88358 & 0.89015 \\
\hline 30 & 0.85108 & 0.85755 & 0.86404 & 0.87054 \\
\hline 31 & 0.84987 & 0.85634 & 0.86282 & 0.86932 \\
\hline 32 & 0.84927 & 0.85574 & 0.86222 & 0.86871 \\
\hline 33 & 0.84907 & 0.85554 & 0.86202 & 0.86851 \\
\hline
\end{tabular}


Table 15. SI index at different PLs (0.9 lag p.f.).

\begin{tabular}{|c|c|c|c|c|}
\hline \multirow{2}{*}{ Bus No } & \multicolumn{4}{|c|}{ SI index } \\
\hline & $P L=0 \%$ & $P L=20 \%$ & $P L=40 \%$ & $P L=60 \%$ \\
\hline $\mathbf{0}$ & 1.00000 & 1.00000 & 1.00000 & 1.00000 \\
\hline 1 & 0.97654 & 0.97738 & 0.97822 & 0.97905 \\
\hline 2 & 0.95664 & 0.95822 & 0.95979 & 0.96135 \\
\hline 3 & 0.92982 & 0.93259 & 0.93534 & 0.93809 \\
\hline 4 & 0.90741 & 0.91121 & 0.91500 & 0.91879 \\
\hline 5 & 0.88661 & 0.89141 & 0.89622 & 0.90102 \\
\hline 6 & 0.87280 & 0.87756 & 0.88231 & 0.88707 \\
\hline 7 & 0.86530 & 0.87003 & 0.87476 & 0.87950 \\
\hline 8 & 0.85646 & 0.86117 & 0.86588 & 0.87059 \\
\hline 9 & 0.85228 & 0.85697 & 0.86167 & 0.86637 \\
\hline 10 & 0.85066 & 0.85535 & 0.86005 & 0.86474 \\
\hline 11 & 0.85018 & 0.85487 & 0.85956 & 0.86426 \\
\hline 12 & 0.95551 & 0.95707 & 0.95863 & 0.96019 \\
\hline 13 & 0.95433 & 0.95589 & 0.95745 & 0.95900 \\
\hline 14 & 0.95401 & 0.95557 & 0.95713 & 0.95868 \\
\hline 15 & 0.95398 & 0.95555 & 0.95711 & 0.95866 \\
\hline 16 & 0.87053 & 0.87528 & 0.88003 & 0.88478 \\
\hline 17 & 0.85728 & 0.86199 & 0.86671 & 0.87142 \\
\hline 18 & 0.84276 & 0.84743 & 0.85210 & 0.85678 \\
\hline 19 & 0.83126 & 0.83591 & 0.84055 & 0.84519 \\
\hline 20 & 0.82135 & 0.82597 & 0.83058 & 0.83520 \\
\hline 21 & 0.81011 & 0.8147 & 0.81928 & 0.82387 \\
\hline 22 & 0.80100 & 0.80555 & 0.81011 & 0.81467 \\
\hline 23 & 0.79249 & 0.79702 & 0.80156 & 0.80609 \\
\hline 24 & 0.78845 & 0.79297 & 0.79749 & 0.80201 \\
\hline 25 & 0.78689 & 0.79141 & 0.79593 & 0.80045 \\
\hline 26 & 0.78643 & 0.79094 & 0.79546 & 0.79998 \\
\hline 27 & 0.87168 & 0.87643 & 0.88119 & 0.88594 \\
\hline 28 & 0.87088 & 0.87562 & 0.88037 & 0.88512 \\
\hline 29 & 0.87047 & 0.87522 & 0.87997 & 0.88472 \\
\hline 30 & 0.85108 & 0.85577 & 0.86047 & 0.86516 \\
\hline 31 & 0.84987 & 0.85456 & 0.85925 & 0.86395 \\
\hline 32 & 0.84927 & 0.85396 & 0.85865 & 0.86334 \\
\hline 33 & 0.84907 & 0.85376 & 0.85845 & 0.86314 \\
\hline
\end{tabular}


Table 16. Power loss of 33-bus at different PLs (lead p.f.).

\begin{tabular}{|c|c|c|c|c|}
\hline \multirow{2}{*}{ Line No } & \multicolumn{4}{|c|}{ Line Loss in $k W$} \\
\hline & $P L=0 \%$ & $P L=20 \%$ & $P L=40 \%$ & $P L=60 \%$ \\
\hline 1 & 31.17 & 27.92 & 24.87 & 22.00 \\
\hline 2 & 25.90 & 23.07 & 20.42 & 17.94 \\
\hline 3 & 35.79 & 31.68 & 27.84 & 24.26 \\
\hline 4 & 29.14 & 25.62 & 22.33 & 19.29 \\
\hline 5 & 25.91 & 22.60 & 19.52 & 16.69 \\
\hline 6 & 6.360 & 6.340 & 6.310 & 6.290 \\
\hline 7 & 2.880 & 2.860 & 2.850 & 2.840 \\
\hline 8 & 2.640 & 2.630 & 2.620 & 2.610 \\
\hline 9 & 0.910 & 0.910 & 0.910 & 0.900 \\
\hline 10 & 0.220 & 0.220 & 0.220 & 0.220 \\
\hline 11 & 0.020 & 0.020 & 0.020 & 0.020 \\
\hline 12 & 0.100 & 0.100 & 0.100 & 0.100 \\
\hline 13 & 0.060 & 0.060 & 0.060 & 0.060 \\
\hline 14 & 0.010 & 0.010 & 0.010 & 0.010 \\
\hline 15 & 0.000 & 0.000 & 0.000 & 0.000 \\
\hline 16 & 13.47 & 13.42 & 13.37 & 13.32 \\
\hline 17 & 10.16 & 10.12 & 10.08 & 10.04 \\
\hline 18 & 10.34 & 10.30 & 10.26 & 10.22 \\
\hline 19 & 7.360 & 7.330 & 7.300 & 7.280 \\
\hline 20 & 5.560 & 5.540 & 5.520 & 5.500 \\
\hline 21 & 5.570 & 5.540 & 5.520 & 5.500 \\
\hline 22 & 3.760 & 3.750 & 3.730 & 3.720 \\
\hline 23 & 2.770 & 2.760 & 2.750 & 2.740 \\
\hline 24 & 0.960 & 0.960 & 0.960 & 0.950 \\
\hline 25 & 0.230 & 0.230 & 0.230 & 0.220 \\
\hline 26 & 0.030 & 0.030 & 0.030 & 0.030 \\
\hline 27 & 0.100 & 0.100 & 0.10 & 0.100 \\
\hline 28 & 0.040 & 0.040 & 0.040 & 0.040 \\
\hline 29 & 0.010 & 0.010 & 0.010 & 0.010 \\
\hline 30 & 0.100 & 0.100 & 0.100 & 0.100 \\
\hline 31 & 0.080 & 0.070 & 0.070 & 0.070 \\
\hline 32 & 0.030 & 0.020 & 0.020 & 0.020 \\
\hline 33 & 0.000 & 0.000 & 0.000 & 0.000 \\
\hline Total kW Loss & 221.68 & 204.36 & 188.17 & 173.09 \\
\hline
\end{tabular}


Table 17. Power loss of 33-bus at different PLs (lag p.f.).

\begin{tabular}{|c|c|c|c|c|}
\hline \multirow{2}{*}{ Line No } & \multicolumn{4}{|c|}{ Line Loss in $k W$} \\
\hline & $P L=0 \%$ & $P L=20 \%$ & $P L=40 \%$ & $P L=60 \%$ \\
\hline 1 & 31.17 & 29.43 & 27.87 & 26.49 \\
\hline 2 & 25.90 & 24.38 & 23.03 & 21.84 \\
\hline 3 & 35.79 & 33.59 & 31.64 & 29.94 \\
\hline 4 & 29.14 & 27.26 & 25.60 & 24.16 \\
\hline 5 & 25.91 & 24.14 & 22.60 & 21.28 \\
\hline 6 & 6.360 & 6.340 & 6.330 & 6.310 \\
\hline 7 & 2.880 & 2.870 & 2.860 & 2.850 \\
\hline 8 & 2.640 & 2.630 & 2.620 & 2.620 \\
\hline 9 & 0.910 & 0.910 & 0.910 & 0.910 \\
\hline 10 & 0.220 & 0.220 & 0.220 & 0.220 \\
\hline 11 & 0.020 & 0.020 & 0.020 & 0.020 \\
\hline 12 & 0.100 & 0.10 & 0.100 & 0.100 \\
\hline 13 & 0.060 & 0.060 & 0.060 & 0.060 \\
\hline 14 & 0.010 & 0.010 & 0.010 & 0.010 \\
\hline 15 & 0.000 & 0.000 & 0.000 & 0.000 \\
\hline 16 & 13.47 & 13.43 & 13.40 & 13.36 \\
\hline 17 & 10.16 & 10.13 & 10.10 & 10.08 \\
\hline 18 & 10.34 & 10.31 & 10.28 & 10.25 \\
\hline 19 & 7.360 & 7.340 & 7.320 & 7.300 \\
\hline 20 & 5.560 & 5.550 & 5.530 & 5.520 \\
\hline 21 & 5.570 & 5.550 & 5.530 & 5.520 \\
\hline 22 & 3.760 & 3.750 & 3.740 & 3.730 \\
\hline 23 & 2.770 & 2.760 & 2.750 & 2.750 \\
\hline 24 & 0.960 & 0.960 & 0.960 & 0.950 \\
\hline 25 & 0.230 & 0.230 & 0.230 & 0.230 \\
\hline 26 & 0.030 & 0.030 & 0.030 & 0.030 \\
\hline 27 & 0.100 & 0.100 & 0.100 & 0.100 \\
\hline 28 & 0.040 & 0.040 & 0.040 & 0.040 \\
\hline 29 & 0.010 & 0.010 & 0.010 & 0.010 \\
\hline 30 & 0.100 & 0.100 & 0.100 & 0.100 \\
\hline 31 & 0.080 & 0.070 & 0.070 & 0.070 \\
\hline 32 & 0.030 & 0.020 & 0.020 & 0.020 \\
\hline 33 & 0.000 & 0.000 & 0.000 & 0.000 \\
\hline Total kW Loss & 221.68 & 212.34 & 204.08 & 196.87 \\
\hline
\end{tabular}


Table 18. Voltage and voltage index of bus-5 in all cases.

\begin{tabular}{|c|c|c|c|c|c|}
\hline Case & & 1 & 2 & 3 & 4 \\
\hline$P L \%$ & & $0 \%$ & $20 \%$ & $40 \%$ & $60 \%$ \\
\hline \multirow[t]{3}{*}{$P^{W T}(k W)$} & & 0.0000 & 258.68 & 517.35 & 776.03 \\
\hline & $V_{\text {Bus-5 }} p . u$ & 0.97042 & 0.97222 & 0.97402 & 0.97581 \\
\hline & $S I_{\text {Bus-5 }}$ & 0.88661 & 0.89324 & 0.89989 & 0.90654 \\
\hline \multirow[t]{5}{*}{0.9 Lead p.f } & $\lambda_{\max }$ & 5.3188 & 5.3433 & 5.3678 & 5.3922 \\
\hline & $P_{\text {Loss }}(k W)$ & 221.68 & 204.36 & 188.17 & 173.09 \\
\hline & PLR\% & $0.00 \%$ & $7.81 \%$ & $15.11 \%$ & $21.91 \%$ \\
\hline & $V_{\text {Bus-5 }} p . u$ & 0.97042 & 0.97173 & 0.97303 & 0.97433 \\
\hline & $S I_{\text {Bus-5 }}$ & 0.88661 & 0.89141 & 0.89622 & 0.90102 \\
\hline \multirow[t]{3}{*}{0.9 Lag. p.f } & $\lambda_{\max }$ & 5.3188 & 5.3359 & 5.3529 & 5.3698 \\
\hline & $P_{\text {Loss }}(k W)$ & 221.68 & 212.34 & 204.08 & 196.87 \\
\hline & PLR\% & $0.00 \%$ & $4.20 \%$ & $7.94 \%$ & $11.20 \%$ \\
\hline
\end{tabular}

Table 19. Optimal location for installing WT on the system.

\begin{tabular}{cc}
\hline WT Optimal Location & Bus 20 \\
\hline Active Poptimal Size & $2.96736 \mathrm{MW}$ \\
Reactive Power loss without $W T$ & $221.72 \mathrm{~kW}$ \\
Active Power loss with $W T$ & $65.04 \mathrm{kVAr}$ \\
Reactive Power loss with $W T$ & $93.74 \mathrm{~kW}$ \\
Minimum Voltage without $W T$ & $25.31 \mathrm{kVAr}$ \\
Maximum Voltage without $W T$ & 0.94170 p.u (Bus 26) \\
Minimum Voltage with $W T$ & 1.00000 p.u (Bus 1) \\
Maximum Voltage with $W T$ & 0.97781 p.u (Bus 33) \\
\end{tabular}

Table 20. Optimal size of WT at various buses.

\begin{tabular}{ccc}
\hline Bus No & Optimal Size of $\boldsymbol{W} \mathbf{T}(\mathbf{M W})$ & System Losses $(\mathbf{k W})$ \\
\hline $\mathbf{0}$ & 4.86 & 221.7 \\
$\mathbf{1}$ & 5.05 & 196.9 \\
$\mathbf{2}$ & 4.87 & 176.6 \\
$\mathbf{3}$ & 4.64 & 149.3 \\
$\mathbf{4}$ & 4.50 & 127.7 \\
$\mathbf{5}$ & 4.31 & 109.1 \\
$\mathbf{6}$ & 3.38 & 121.2 \\
$\mathbf{7}$ & 2.97 & 127.1 \\
$\mathbf{8}$ & 2.55 & 135.0 \\
$\mathbf{9}$ & 2.32 & 140.3 \\
$\mathbf{1 0}$ & 2.18 & 144.0 \\
$\mathbf{1 1}$ & 2.09 & 147.5 \\
$\mathbf{1 2}$ & 2.97 & 193.2 \\
$\mathbf{1 3}$ & 1.99 & 202.2 \\
$\mathbf{1 4}$ & 1.72 & 204.8 \\
\hline
\end{tabular}




\begin{tabular}{llll} 
Continued & & & \\
\hline $\mathbf{1 5}$ & 1.58 & 206.0 \\
$\mathbf{1 6}$ & 3.94 & 103.1 \\
$\mathbf{1 7}$ & 3.66 & 99.00 \\
$\mathbf{1 8}$ & 3.38 & 95.50 \\
$\mathbf{1 9}$ & 3.15 & 93.90 \\
$\mathbf{2 0}$ & $\mathbf{2 . 9 7}$ & $\mathbf{9 3 . 7 0}$ \\
$\mathbf{2 1}$ & 2.74 & 95.20 \\
$\mathbf{2 2}$ & 2.55 & 98.10 \\
$\mathbf{2 3}$ & 2.32 & 102.90 \\
$\mathbf{2 4}$ & 2.18 & 107.10 \\
$\mathbf{2 5}$ & 2.09 & 110.50 \\
$\mathbf{2 6}$ & 2.04 & 113.70 \\
$\mathbf{2 7}$ & 2.97 & 132.60 \\
$\mathbf{2 8}$ & 2.64 & 141.80 \\
$\mathbf{2 9}$ & 2.36 & 149.60 \\
$\mathbf{3 0}$ & 2.13 & 145.50 \\
$\mathbf{3 1}$ & 1.95 & 151.70 \\
$\mathbf{3 2}$ & 1.81 & 155.90 \\
& 1.76 & 158.50 \\
\hline
\end{tabular}

\section{Conclusion}

The paper studied the impact of installing wind turbine in radial distribution system with different PLs on voltage stability and power loss reduction, the optimum size and location of wind turbine are also determined in this paper. The study is applied on the 9-bus and 33-bus test systems. The results show that as penetration level increases voltage stability and power loss reduction are enhanced especially with lead p.f. operation. Voltage stability index reflects a guide to choose the suitable bus for installing wind turbines DG, and these results are supported by PLR and load margin of the system.

\section{References}

[1] European Wind Energy Association (2009) Wind Energy Facts. Eartscan, London.

[2] Committee on Environmental Impacts of Wind Energy Projects (2007) Environmental Impacts of Wind Energy Projects. The National Academies Press, Washington DC.

[3] Masannagari, A.R. (2008) Optimization the Size and Location of Distributed Generators to Maximize the Grid Stability. Master of Science, Mississippi State University, Starkville.

[4] Hemdan, N.G.A. and Kurrat, M. (2008) Distributed Generation Location and Capacity Effect on Voltage Stability of Distribution Networks. Annual IEEE Conference Student Paper, Aalborg, 15-26 February 2008, 1-5.

[5] Thong, V.V., Driesen, J. and Belmans, R. (2007) Benefits and Impact of Using Small Generators for Network Support. IEEE Power Engineering Society General Meeting, Tampa, FL, $24-28$ June 2007, 1-7.

[6] Chen, H.Y., Chen, J.F., Shi, D.Y. and Duan, X.Z. (2006) Power Flow Study and Voltage Stability Analysis for Distribution Systems with Distributed Generation. IEEE Power Engineering Society General Meeting, Montreal.

[7] Le, H.T. and Santoso, S. (2007) Analysis of Voltage Stability and Optimal Wind Power Penetration Limits for a Non-radial Network with an Energy Storage System. IEEE Power Engineering Society General Meeting, Tampa, FL, 24-28 June 2007, 1-8.

[8] El-Saadawi, M.M., Kaddah, S.S., Osman, M.G. and Abdel-Wahab, M.N. (2008) Impact of Wind Farms on Contingent Power System Voltage Stability. 12th International Middle-East Power System Conference, Aswan, 12-15 March 2008, 637-644.

[9] AlHajri, M. (2009) Sizing and Placement of Distributed Generation in Electrical Distribution Systems using Conventional and Heuristic Optimization Methods. Doctor of Philosophy, Dalhousie University, Nova Scotia. 
[10] Thukaram, D., Wijekoon Banda, H.M. and Jerome, J. (1999) A Robust Three Phase Power Flow Algorithm for Radial Distribution Systems. Electric Power Systems Research, 50, 227-236.

[11] Abu-Mouti, F.S. (2008) Radial Distribution Feeders Compensation Using Distributed Generation. Master of Applied Science, Dalhousie University, Nova Scotia.

[12] Khaniya, D. (2008) Development of Three Phase Continuation Power Flow for Voltage Stability Analysis of Distribution System. Degree of Master of Science, Mississippi State University, Starkville.

[13] Verbič, G., Pantoš, M. and Gubina, F. (2006) On Voltage Collapse and Apparent-Power Losses. Electric Power Systems Research, 76, 760-767. http://dx.doi.org/10.1016/j.epsr.2005.10.007

[14] IEEE/CIGRE Joint Task Force on Stability Terms and Definitions (2004) Definition and Classification of Power System Stability. IEEE Transactions on Power Systems, 19, 1387-1401.

[15] Chakravorty, M. and Das, D. (2001) Voltage Stability Analysis of Radial Distribution Networks. Electrical Power and Energy Systems, 23, 129-135. http://dx.doi.org/10.1016/S0142-0615(00)00040-5

[16] Rao, S.S. (1996) Engineering Optimization: Theory and Practice. 3rd Edition, Wiley-Interscience, New York.

[17] Goswami, S.K., Ghose, T. and Basu, S.K. (1999) An Approximate Method for Capacitor Placement in Distribution System Using Heuristics and Greedy Search Technique. Electric Power Systems Research, 51, 143-151. http://dx.doi.org/10.1016/S0378-7796(98)00166-7

[18] Gözel, T., Eminoglu, U. and Hocaoglu, M.H. (2008) A Tool for Voltage Stability and Optimization (VS\&OP) in Radial Distribution Systems Using Matlab Graphical User Interface (GUI). Simulation Modelling Practice and Theory, 16, 505-518. http://dx.doi.org/10.1016/j.simpat.2008.02.003

[19] Srinivasa Rao, R. (2010) Optimal Capacitor Allocation for loss reduction in Distribution System Using Fuzzy and Plant Growth Simulation Algorithm. International Journal of Computer, Electrical, Automation, Control and Information Engineering, 4, 1236-1242. 


\section{Appendices}

\section{Appendix A}

Power flow solution

\section{A). Power Flow Solution for Single Line Feeders}

Using the system in Figure A1, the solution steps are summarized as follows:

1). Find the sum of active and reactive power loads for all buses as well as the sum of all resistances and inductive reactances of each section connecting two buses.

2). Assume that sending end real power, reactive power and voltage to be approximated by:

$$
\begin{aligned}
& P o=\sum_{i=0}^{n} P_{L_{i+1}}+P_{\text {factor }} \\
& Q o=\sum_{i=0}^{n} Q_{L_{i+1}}+Q_{\text {factor }} \\
& \mathrm{V}_{0}=1+\mathrm{j} 0 \text { p.u }
\end{aligned}
$$

where the incremental increases $P_{\text {factor }}$ and $Q_{\text {factor }}$ are given by:

$$
\begin{gathered}
P_{\text {factor }}=\left[\frac{\left(\left(\sum_{i=0}^{n} P_{L_{i+1}}\right)^{2}+\left(\sum_{i=0}^{n} Q_{L_{i+1}}\right)^{2}\right) / V o^{2}}{n-1}\right] \times \sum_{i=0}^{n} r_{i+1} \\
Q_{\text {factor }}=\left[\frac{\left(\left(\sum_{i=0}^{n} P_{L_{i+1}}\right)^{2}+\left(\sum_{i=0}^{n} Q_{L_{i+1}}\right)^{2}\right) / V o^{2}}{n-1}\right] \times \sum_{i=0}^{n} x_{i+1}
\end{gathered}
$$

Equation (4-A) and (5-A) are due to the fact that in reality, there are no lossless systems and there is rarely a feeder with only two buses. These approximation factors are added to reduce the required number of iterations required for a solution i.e., get closer to the exact loss values, then, use these values $\mathrm{P}_{0} \& Q_{0}$ in the initial iteration.

$3)$. Apply the following power flow equations to the feeder.

$$
\begin{aligned}
P_{i+1} & =P_{i}-\left[r_{i+1}\left(P_{I}^{2}+Q_{i}^{2}\right) / V_{i}^{2}\right]-P_{L_{i+1}}+\mu_{P} A P_{i+1} \\
Q_{i+1} & =Q_{i}-\left[x_{i+1}\left(P_{I}^{2}+Q_{i}^{2}\right) / V_{i}^{2}\right]-Q_{L_{i+1}}+\mu_{0} R P_{i+1} \\
V_{i+1}^{2} & =V_{i}^{2}-2\left(r_{i+1} P_{i}+x_{i+1} Q_{i}\right)+\left[r_{i+1}^{2}+x_{i+1}^{2}\left(P_{i}^{2}+Q_{i}^{2}\right) / V_{i}^{2}\right]
\end{aligned}
$$

where:

$P_{\text {factor: }}$ : Real power loss approximation.

$Q_{\text {factor: }}$ : Reactive power loss approximation.

$\mu_{p}$ : Active power multiplier, set to zero when there is no active power source or set to 1 when there is active power source.

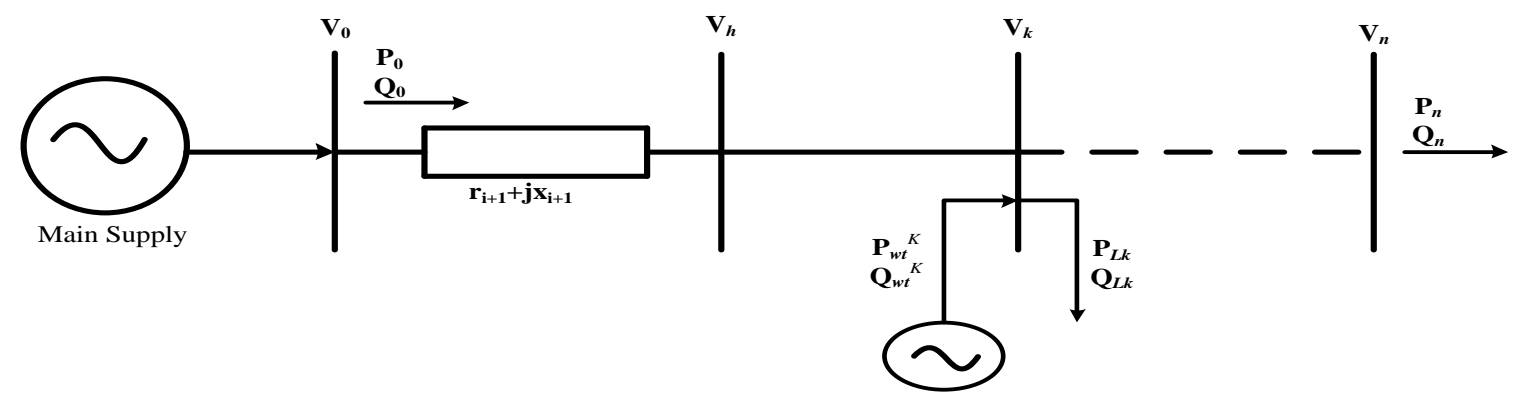

Figure A1. Radial distribution feeder model including wind turbine. 
$\mu_{0}$ : Reactive power multiplier, set to zero when there is no reactive power source or set to \pm 1 when there is a reactive power source.

$A P_{i+1}$ :Active Power magnitude injected at bus $i+1$

$R P_{i+1}:$ Reactive Power magnitude injected at bus $i+1$

4). If the absolute values of $P n \& Q n$ of the last bus are zero or within an acceptable tolerance, the power flow solution is acceptable. Otherwise, go to the next step.

5). For the first bus in the main feeder set:

$$
\begin{gathered}
P_{O_{\text {new }}}=P_{O_{\text {old }}}+P_{n} \\
Q_{O_{\text {new }}}=Q_{O_{\text {old }}}+Q_{n}
\end{gathered}
$$

Then, use these new initial values and repeat step 3.

A). Power Flow Solution for Lateral and Sub-lateral Feeders:

1). Find the sum of real and reactive power loads on the sub-laterals (if available) and represent them as loads on their laterals. Do the same for laterals and represent them as loads on the main feeder.

2). Apply the recursive power flow solution algorithm as in (single line feeders) for the main feeder.

$3)$. Set the voltage of the far bus that represents a lateral to be equal to the $\left(V_{0}\right)$ of this lateral. Solve the lateral individually as described in the case of the single line feeder.

4). For sub-laterals (if available), set the voltage of the far bus that represents a sub-lateral to be equal to the $\left(V_{0}\right)$ of this sub-lateral. Solve the sub-lateral individually as described in the case of the single line feeder.

5). Get the total real and reactive powers injected into the sub-lateral and represent them again as a single load on its lateral.

6). Apply the power flow solution to the lateral individually again. If there is another sub-lateral go back to step 4 and solve the second far bus on the lateral that represents the sub-lateral. Otherwise, go to next step.

7). Find the total real and reactive powers injected into the lateral (use data from last power flow run) and represent them again as a load on the main feeder.

8). Apply the power flow recursion to the main feeder. If there are more laterals, go back to step 3 and solve for the second far bus that represents the lateral on the main feeder. If not, go to the next step.

9). With the bus voltages found using the previous step, solve the laterals and sub-laterals (if available) individually. Then the power flow solution is reached. 


\section{Appendix B}

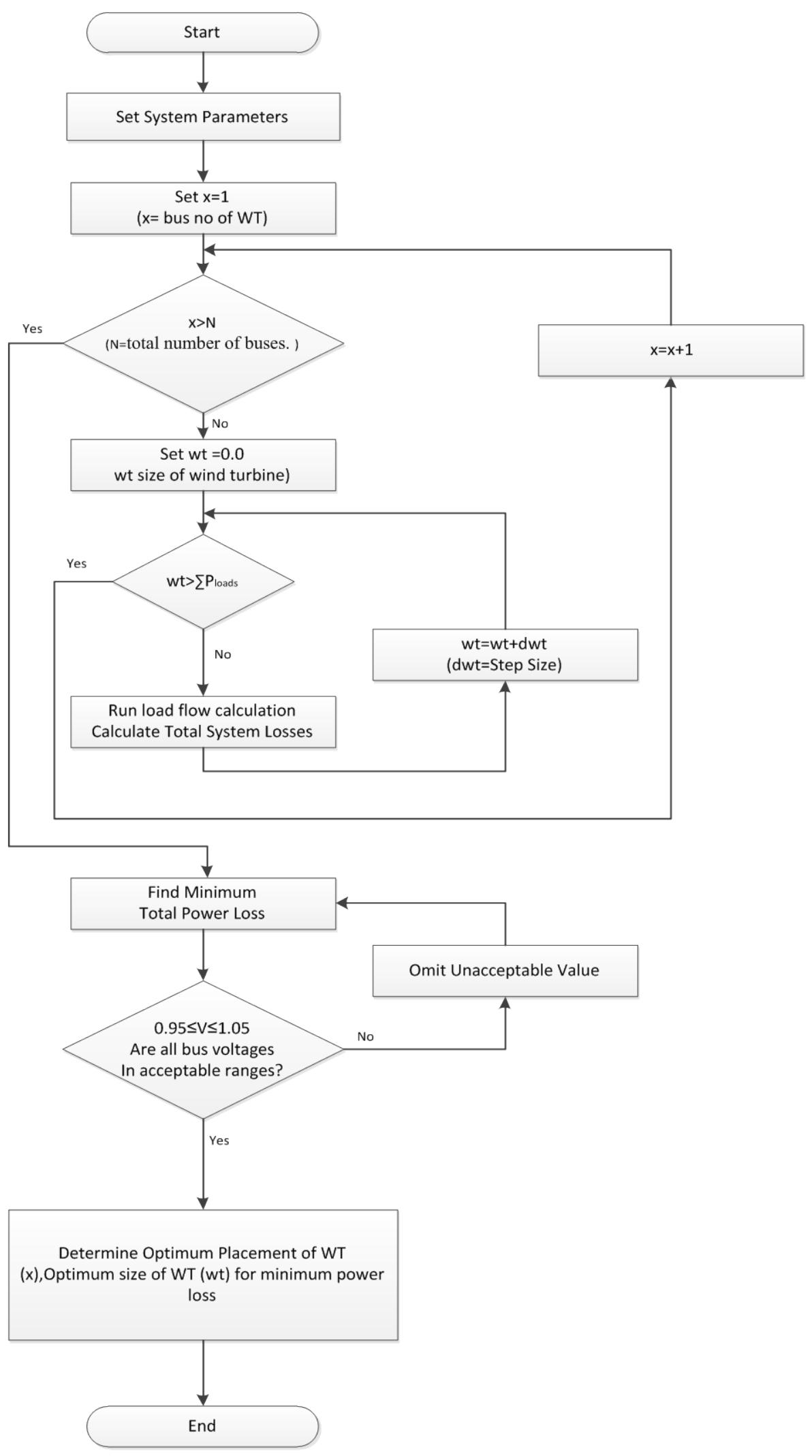

Figure B-1. Flowchart of the grid search algorithm. 


\section{Appendix C}

Table C-1. 9-Bus feeder systems data.

\begin{tabular}{ccccccc}
\hline Line No & From Bus, $i$ & To Bus, $i+1$ & $\mathrm{R}_{i, i+1} \Omega$ & $\mathrm{X}_{i, i+1} \Omega$ & $\mathrm{P}_{\mathrm{L} i+1}(\mathrm{KW})$ & $\mathrm{Q}_{\mathrm{L} i+1}(\mathrm{KVAr})$ \\
\hline 1 & 0 & 1 & 0.1233 & 0.4127 & 1840 & 460 \\
2 & 1 & 2 & 0.014 & 0.6057 & 980 & 340 \\
3 & 2 & 3 & 0.7463 & 1.205 & 1790 & 446 \\
4 & 3 & 4 & 0.6984 & 0.6084 & 1598 & 1840 \\
5 & 4 & 5 & 1.9831 & 1.7276 & 1610 & 600 \\
6 & 5 & 6 & 0.9053 & 0.7886 & 780 & 110 \\
7 & 6 & 7 & 2.0552 & 1.164 & 1150 & 60 \\
8 & 7 & 8 & 4.7953 & 2.716 & 980 & 130 \\
9 & 8 & 9 & 5.3434 & 3.0264 & 1640 & 200 \\
\hline
\end{tabular}

Table C-2. 33-Bus feeder systems data.

\begin{tabular}{|c|c|c|c|c|c|c|}
\hline Line No & From Bus, $i$ & To Bus $i+1$ & $\mathrm{R}_{i, i+1} \Omega$ & $\mathrm{X}_{i, i+1} \Omega$ & $\mathrm{P}_{\mathrm{L} i+1} \mathrm{~kW}$ & $\mathrm{Q}_{\mathrm{L} i+1} \mathrm{kVAr}$ \\
\hline 1 & 0 & 1 & 0.1170 & 0.0480 & 230 & 142.5 \\
\hline 2 & 1 & 2 & 0.1073 & 0.0440 & 0 & 0 \\
\hline 3 & 2 & 3 & 0.1645 & 0.0457 & 230 & 142.5 \\
\hline 4 & 3 & 4 & 0.1495 & 0.0415 & 230 & 142.5 \\
\hline 5 & 4 & 5 & 0.1495 & 0.0415 & 0 & 0 \\
\hline 6 & 5 & 6 & 0.3144 & 0.0540 & 0 & 0 \\
\hline 7 & 6 & 7 & 0.2096 & 0.0360 & 230 & 142.5 \\
\hline 8 & 7 & 8 & 0.3144 & 0.0540 & 230 & 142.5 \\
\hline 9 & 8 & 9 & 0.2096 & 0.0360 & 0 & 0 \\
\hline 10 & 9 & 10 & 0.1310 & 0.0225 & 230 & 142.5 \\
\hline 11 & 10 & 11 & 0.1048 & 0.0180 & 137 & 84 \\
\hline 12 & 11 & 12 & 0.1572 & 0.0270 & 72 & 45 \\
\hline 13 & 12 & 13 & 0.2096 & 0.0360 & 72 & 45 \\
\hline 14 & 13 & 14 & 0.1048 & 0.0180 & 72 & 45 \\
\hline 15 & 14 & 15 & 0.0524 & 0.0090 & 13.5 & 7.5 \\
\hline 16 & 15 & 16 & 0.1794 & 0.0489 & 230 & 142.5 \\
\hline 17 & 16 & 17 & 0.1645 & 0.0457 & 230 & 142.5 \\
\hline 18 & 17 & 18 & 0.2079 & 0.0473 & 230 & 142.5 \\
\hline 19 & 18 & 19 & 0.1890 & 0.0430 & 230 & 142.5 \\
\hline 20 & 19 & 20 & 0.1890 & 0.0430 & 230 & 142.5 \\
\hline 21 & 20 & 21 & 0.2620 & 0.0450 & 230 & 142.5 \\
\hline 22 & 21 & 22 & 0.2620 & 0.0450 & 230 & 142.5 \\
\hline 23 & 22 & 23 & 0.3144 & 0.0540 & 230 & 142.5 \\
\hline 24 & 23 & 24 & 0.2096 & 0.0360 & 230 & 142.5 \\
\hline 25 & 24 & 25 & 0.1310 & 0.0225 & 230 & 142.5 \\
\hline 26 & 25 & 26 & 0.1048 & 0.0180 & 137 & 85 \\
\hline 27 & 26 & 27 & 0.1572 & 0.0270 & 75 & 48 \\
\hline 28 & 27 & 28 & 0.1572 & 0.0270 & 75 & 48 \\
\hline 29 & 28 & 29 & 0.1572 & 0.0270 & 75 & 48 \\
\hline 30 & 29 & 30 & 0.1572 & 0.0270 & 57 & 34.5 \\
\hline 31 & 30 & 31 & 0.2096 & 0.0360 & 57 & 34.5 \\
\hline 32 & 31 & 32 & 0.1572 & 0.0270 & 57 & 34.5 \\
\hline 33 & 32 & 33 & 0.1048 & 0.0180 & 57 & 34.5 \\
\hline
\end{tabular}

